\title{
Phenomenology of single-inclusive jet production with jet radius and threshold resummation
}

\author{
Xiaohui Liu* \\ Center of Advanced Quantum Studies, Department of Physics, Beijing Normal University, \\ Beijing 100875, China \\ Sven-Olaf Moch ${ }^{\dagger}$ \\ II. Institut für Theoretische Physik, Universität Hamburg, Luruper Chaussee 149, \\ D-22761 Hamburg, Germany \\ Felix Ringer \\ Nuclear Science Division, Lawrence Berkeley National Laboratory, Berkeley, California 94720, USA
}

(Received 7 February 2018; published 30 March 2018)

\begin{abstract}
We perform a detailed study of inclusive jet production cross sections at the LHC and compare the QCD theory predictions based on the recently developed formalism for threshold and jet radius joint resummation at next-to-leading logarithmic accuracy to inclusive jet data collected by the CMS Collaboration at $\sqrt{S}=7$ and $13 \mathrm{TeV}$. We compute the cross sections at next-to-leading order in QCD with and without the joint resummation for different choices of jet radii $R$ and observe that the joint resummation leads to crucial improvements in the description of the data. Comprehensive studies with different parton distribution functions demonstrate the necessity of considering the joint resummation in fits of those functions based on the LHC jet data.
\end{abstract}

DOI: 10.1103/PhysRevD.97.056026

\section{INTRODUCTION}

Long-term persistence in achieving higher-order calculations in perturbative quantum chromodynamics (pQCD) paves the way to the precision frontier at the Large Hadron Collider (LHC). With many standard model processes now being measured with an impressive accuracy at the LHC, theoretical predictions beyond next-to-leading order (NLO) in $\mathrm{pQCD}$, nowadays considered the standard in phenomenological analyses, are often mandatory. During the past three years, there has been a burst of publications on complete next-to-next-to-leading-order (NNLO) calculations for various hadro-production processes involving jets [1-9]. The list of those processes includes the hadroproduction of gauge bosons $V+$ jet $\left(V=W^{ \pm}, Z, \gamma\right)$ as well as single-inclusive jets and dijets, but it is limited to $2 \rightarrow 2$ reactions at the Born level due to the enormous computational complexity at NNLO. In particular, the calculations for

\footnotetext{
*xiliu@bnu.edu.cn

sven-olaf.moch@desy.de

fmringer@lbl.gov
}

Published by the American Physical Society under the terms of the Creative Commons Attribution 4.0 International license. Further distribution of this work must maintain attribution to the author(s) and the published article's title, journal citation, and DOI. Funded by SCOAP.
$V+$ jet production have already been shown to greatly improve the description of the available LHC data [10-12].

For the hadro-production of jets at the LHC, the experimental collaborations have provided very precise data for the single-inclusive jet production cross sections $p p \rightarrow$ jet $+X$ at all collider energies and differential in the jet transverse momentum $p_{T}$ and the rapidity $\eta$. Specifically, ALICE [13], ATLAS [14] and CMS [15] have collected data at $\sqrt{S}=2.76 \mathrm{TeV}$ and ATLAS and CMS at $\sqrt{S}=7 \mathrm{TeV}$ $[16,17], 8 \mathrm{TeV}[18,19]$ and $13 \mathrm{TeV}$ [20-22]. These data allow for important consistency tests of $\mathrm{pQCD}$ as well as a precise extraction of the value of the strong coupling constant $\alpha_{s}\left(M_{Z}\right)$ [23], and they provide very valuable constraints on parton distribution functions (PDFs) which govern the parton luminosity of the colliding initial protons [24,25].

In order to fully utilize the available data, a precise understanding of the corresponding theoretical calculations within $\mathrm{pQCD}$ is very important. The current accuracy for fixed-order $\mathrm{pQCD}$ predictions is NNLO where the $\alpha_{s}^{2}$ coefficient is known in the leading-color approximation [7], i.e., for large values of $N_{c}$ for a general $\mathrm{SU}\left(N_{c}\right)$ gauge group. Any additional corrections are parametrically suppressed as $1 / N_{c}^{2}$, so that the results of [7] are supposed to approximate the full NNLO calculation very well. Preliminary comparisons of those NNLO results with some of the LHC data, however, have not been entirely satisfactory. 
References [21,26] have shown that the agreement between theory and data heavily depends on the choices for the renormalization and factorization scales $\mu_{R}$ and $\mu_{F}$. Moreover, for some natural scale choices, such as identifying $\mu_{R}$ and $\mu_{F}$ with the transverse momentum $p_{T}^{\max }$ of the leading jet in the event, i.e., $\mu_{R}=\mu_{F}=p_{T}^{\max }$, the theory description of the data at NNLO deteriorates compared to NLO. This situation implies the existence of potentially large higherorder corrections beyond fixed NNLO.

Improvements beyond fixed order in $\mathrm{pQCD}$ are possible by supplementing the fixed-order calculations with resummation results where dominant classes of logarithmic corrections are summed up to all orders in the strong coupling constant. Recently, a joint resummation framework was developed [27] that allows us to resum both threshold and jet radius logarithms simultaneously. Threshold logarithms appear in the partonic cross section at $n$th order as $\alpha_{s}^{n}\left(\ln ^{k}(z) / z\right)_{+}$, where $z=s_{4} / s$ and $k \leq 2 n-1$. Here, $s_{4}$ is the invariant mass of the partonic system recoiling against the observed jet and $s$ is the partonic center-of-mass energy [28]. Since these logarithms are integrated over the specified parton kinematics together with the steeply falling parton luminosity, threshold logarithms can dominate the entire cross section in a wide kinematic range. Instead, the jet radius $R$ is an external quantity and the dependence of the cross section is singlelogarithmic $\alpha_{s}^{n} \ln ^{k}(R)$ with $k \leq n$ instead of double-logarithmic [29-31]. The framework developed in [27] addresses both these logarithmic corrections on the same footing, and it was shown that numerically the threshold and the jet radius logarithmic terms do account for the dominant bulk of the NLO corrections. The explicit resummation of these logarithms to next-to-leading logarithmic (NLL) accuracy was also realized within the joint resummation framework derived in [27], and the subsequent matching to fixed-order NLO results leads to theory predictions at the combined NLO + NLL accuracy. The approach of [27], however, is not limited to this logarithmic accuracy, and the framework is ready for a systematic extension to the next-to-next-to-leading logarithmic (NNLL) accuracy which may then be matched to the available fixed-order NNLO results to achieve a combined accuracy of NNLO + NNLL. We leave the extension to NNLL for future work and instead focus here on the phenomenological results at NLO + NLL accuracy.

In general, one expects competing effects from threshold and small- $R$ resummation. As it was observed in [32-35], threshold resummation leads to an enhancement, whereas small- $R$ resummation alone leads to a decrease of the cross section [30,36]; see also [37] for studies on jet angularities. Depending on the nontrivial interplay within the joint resummation framework, one or the other effect will dominate. For certain kinematics and values of $R$, the two effects may even largely cancel out. In order to obtain a good understanding of the convergence of the perturbative series expansion, it is important to disentangle these two effects. A closely related issue is the dependence of the fixed-order and the resummed calculations on the renormalization and factorization scales $\mu_{R}$ and $\mu_{F}$, collectively denoted by $\mu$ in the following. As it was pointed out in $[21,26]$, the fixedorder results change significantly depending on whether the hard scale is chosen as $\mu=p_{T}$ of the individual jet or as the transverse momentum $p_{T}^{\max }$ of the leading jet in the event. At the same time, the residual scale dependence is very small and even vanishes for some kinematic configurations. In $[29,30,36]$, it was argued that this is generally an artifact of results at fixed order in perturbation theory. Here, we address this issue within the joint resummation formalism.

In this work, we provide a detailed comparison with LHC data and find that the inclusion of the resummation generally yields a much better description of those data. In addition, our studies highlight possible improvements that can be obtained by using a resummed calculation in fits of PDFs. The constraints from inclusive jet data on PDF fits are most significant for the gluon PDF $g(x)$ in the large- $x$ region. In this endpoint region, the cross sections from which PDFs are extracted can be subject to large logarithmic corrections that need to be taken into account to all orders. Improvements in the precision of the extracted PDFs eventually have direct impact on all PDF sensitive analyses at the LHC and recent progress on PDFs in the large- $x$ region has been made in $[38,39]$.

The remainder of this work is organized as follows. In Sec. II, we briefly review the theoretical framework of [27]. In Sec. III, we present detailed phenomenological studies of the resummation effect and the scale dependence of the resummed cross section. We study cross section ratios for different jet radii to discriminate the predictive power of the NLL + NLO and the NLO results. Finally, we present a comprehensive comparison to the inclusive jet data from the LHC together with the impact of different PDF sets. We conclude in Sec. IV with a summary and an outlook.

\section{THEORETICAL FRAMEWORK}

First we review the theoretical formalism which allows us to achieve the threshold and small- $R$ joint resummation used in this work. The resummation is based on the factorization theorem [27] developed within the framework of the soft collinear effective theory [40-43], in which the single-inclusive jet cross section with jet transverse momentum $p_{T}$, jet rapidity $y$ and a small anti- $k_{T}$ [44] jet radius $R$ near the partonic threshold can be written as

$$
\begin{aligned}
\frac{p_{T}^{2} \mathrm{~d}^{2} \sigma}{\mathrm{d} p_{T}^{2} \mathrm{~d} y}= & \sum_{i_{1} i_{2}} \int_{0}^{V(1-W)} \mathrm{d} z \int_{\frac{V W}{1-z}}^{1-\frac{1-V}{1-z}} \mathrm{~d} v x_{1}^{2} f_{i_{1}}\left(x_{1}\right) x_{2}^{2} f_{i_{2}}\left(x_{2}\right) \\
& \times \frac{\mathrm{d}^{2} \hat{\sigma}_{i_{1} i_{2}}}{\mathrm{~d} v \mathrm{~d} z}\left(v, z, p_{T}, R\right),
\end{aligned}
$$

where the partonic cross sections $\hat{\sigma}_{i_{1} i_{2}}$ are further factorized as 


$$
\begin{aligned}
\frac{\mathrm{d}^{2} \hat{\sigma}_{i_{1} i_{2}}}{\mathrm{~d} v \mathrm{~d} z}= & s \int \mathrm{d} s_{X} \mathrm{~d} s_{c} \mathrm{~d} s_{G} \delta\left(z s-s_{X}-s_{G}-s_{c}\right) \\
& \times \operatorname{Tr}\left[\mathbf{H}_{i_{1} i_{2}}\left(v, p_{T}, \mu_{h}, \mu\right) \mathbf{S}_{G}\left(s_{G}, \mu_{s G}, \mu\right)\right] J_{X}\left(s_{X}, \mu_{X}, \mu\right) \\
& \times \sum_{m} \operatorname{Tr}\left[J_{m}\left(p_{T} R, \mu_{J}, \mu\right) \otimes_{\Omega} S_{c, m}\left(s_{c} R, \mu_{s c}, \mu\right)\right] .
\end{aligned}
$$

In Eq. (1), the PDFs are denoted by $f_{i}$ which are evaluated at the momentum fractions $x_{1}=V W / v /(1-z)$ and $x_{2}=$ $(1-V) /(1-v) /(1-z)$, where $V=1-p_{T} e^{-y} / \sqrt{S}, V W=$ $p_{T} e^{y} / \sqrt{S}$ and $\sqrt{S}$ is the hadronic center-of-mass energy. The sum $i$ runs over all partonic channels initiating the subprocesses and $m$ runs over the collinear splitting history. The associated angular integrals are denoted by ' $\otimes_{\Omega}$ ' [45] to resum nonglobal logarithms [45-50]. Besides the jet $p_{T}$, the partonic cross sections depend on the partonic kinematic variables $s=x_{1} x_{2} S$, $z$ and $v=u /(u+t)$ with $t=\left(p_{1}-p_{3}\right)^{2}$ and $u=\left(p_{2}-p_{3}\right)^{2}$. Here, $p_{1,2}$ are the momenta of the incoming partons and $p_{3}$ is the momentum of the parton which initiates the signal-jet.

The $2 \rightarrow 2$ hard scattering functions in Eq. (2) are denoted by $\mathbf{H}_{i_{1} i_{2}}$ which are available to two loops [51]. The inclusive jet function $J_{X}\left(s_{X}\right)$ is also known to order $\alpha_{s}^{2}$ $[52,53]$ and the NLO jet function can be extracted from $[54,55]$. The global soft function $\mathbf{S}_{G}$ and the soft collinear $[45,56]$ function $S_{c}$ have been derived to NLO in $[27,57]$. The global soft function and the soft collinear function can be readily calculated to two loops following [58] and $[59,60]$. All the functions are evolved from their natural scales $\mu_{i}$ to the common scale $\mu$ according to their renormalization group equations in order to obtain the NLL resummation used in this work.

The factorization formalism in Eq. (2) holds in the threshold regime in which $z \rightarrow 1$ and $R \ll 1$. To extend the region of validity, we combine the NLL resummed results with the NLO predictions using an additive matching procedure and define

$$
\sigma_{\mathrm{NLO}+\mathrm{NLL}}=\sigma_{\mathrm{NLO}}-\sigma_{\mathrm{NLO}_{\text {sing }}}+\sigma_{\mathrm{NLL}} .
$$

Here, the logarithmically enhanced contributions at NLO are obtained within the resummation framework and denoted by $\sigma_{\mathrm{NLO}_{\text {sing }}}$. They are subtracted from the full NLO calculation and replaced by the NLL resummed results $\sigma_{\mathrm{NLL}}$. For the

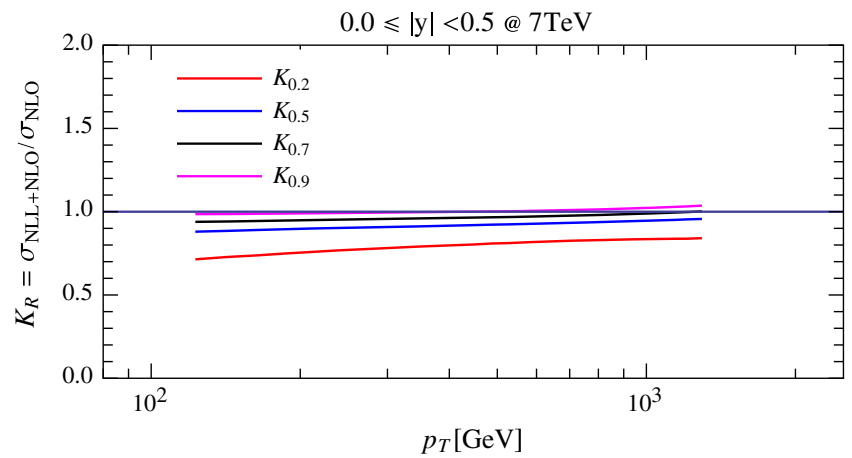

phenomenological studies presented in the next section, we use as a default scale choice the leading jet transverse momentum $\mu_{R}=\mu_{F}=p_{T}^{\max }$ for the fixed NLO calculations [61]. We vary the scales around the central scale up and down by a factor of two and take the maximal deviation as our NLO scale uncertainties. For the resummed results, we make the central scale choices $\mu=\mu_{h}=p_{T}^{\max }, \mu_{J}=p_{T}^{\max } R$ for the hard and the signal-jet functions, respectively, and we set $\mu_{X}=p_{T}^{\max }\left(1-2 p_{T}^{\max } / \sqrt{S}\right)$, see also $[62,63]$. The other scales are determined in the seesaw way: $\mu_{s G}=\mu_{X}^{2} / \mu_{h}$ and $\mu_{s c}=$ $\mu_{J} \times \mu_{s G} / \mu_{h}$ for the global soft and the soft collinear functions, respectively. Our uncertainty estimates are obtained by varying $\mu, \mu_{h}, \mu_{J}$ and $\mu_{X}$ independently by a factor of two around their central values while keeping the seesaw relations for $\mu_{s G}$ and $\mu_{s c}$ in terms of $\mu_{X}$. The final scale uncertainty is obtained by taking the envelope of the scale variations.

\section{PHENOMENOLOGY}

We start by studying the overall numerical impact of the joint threshold and small- $R$ resummation. We then continue by analyzing the scale dependence of the resummed cross section and provide a detailed comparison to LHC data. Finally, we study in detail the impact of different PDF sets. The two single-inclusive jet data sets from CMS that we are comparing to throughout this section were taken at $\sqrt{S}=$ $7 \mathrm{TeV}$ [17] and at $13 \mathrm{TeV}$ [22]. For the $\sqrt{S}=7 \mathrm{TeV}$ data set, the jets were reconstructed using two different values of the jet radius, $R=0.5$ and $R=0.7$ covering a rapidity range of $|y|<3$. Instead, for the $\sqrt{S}=13 \mathrm{TeV}$ data set, the jet radius parameters were chosen as $R=0.4$ and $R=0.7$ covering $|y|<4$.7. For both data sets, the jets were reconstructed using the anti- $k_{T}$ algorithm [44] and the transverse momentum of the identified jets ranges up to $p_{T}=2 \mathrm{TeV}$.

\section{A. Numerical impact of the joint resummation}

In Fig. 1, we plot the ratio $K_{R}$,

$$
K_{R}=\frac{\sigma_{\mathrm{NLL}+\mathrm{NLO}}(R)}{\sigma_{\mathrm{NLO}}(R)}
$$

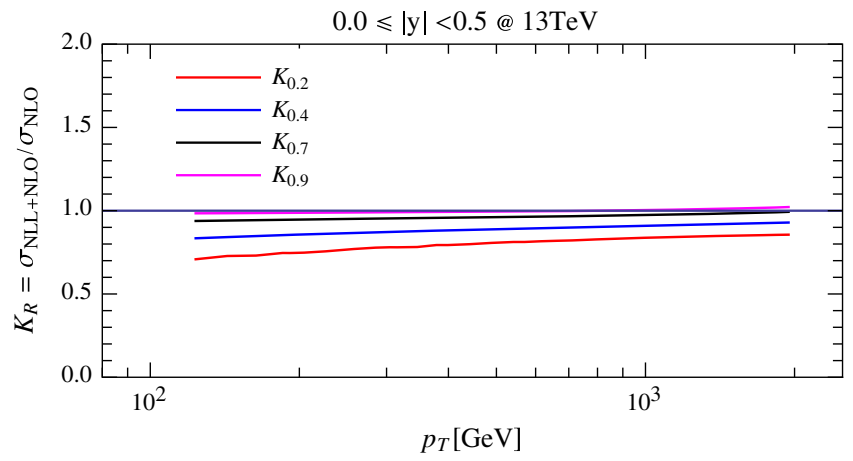

FIG. 1. Cross section ratios $K_{R}$ of Eq. (4) with different jet radii at $\sqrt{S}=7 \mathrm{TeV}$ (left) and $13 \mathrm{TeV}$ (right) using the MMHT PDF set [64] at NLO. 
TABLE I. Cross section ratios $K_{R}$ of Eq. (4) for different jet radii at $\sqrt{S}=7 \mathrm{TeV}$ (top) and $13 \mathrm{TeV}$ (bottom) for selected values of the signal jet $p_{T}$ using the MMHT PDF set [64] at NLO.

\begin{tabular}{|c|c|c|c|c|c|}
\hline$p_{T}[\mathrm{GeV}]$ & 200 & & 300 & 500 & 1000 \\
\hline \multicolumn{6}{|c|}{$0 \leq|y|<0.5, \sqrt{S}=7 \mathrm{TeV}$} \\
\hline$K_{0.2}$ & 0.76 & & 0.78 & 0.81 & 0.83 \\
\hline$K_{0.5}$ & 0.90 & & 0.90 & 0.92 & 0.94 \\
\hline$K_{0.7}$ & 0.95 & & 0.96 & 0.96 & 0.98 \\
\hline$K_{0.9}$ & 0.98 & & 0.99 & 1.00 & 1.02 \\
\hline$p_{T}[\mathrm{GeV}]$ & 200 & 300 & 500 & 1000 & 1500 \\
\hline \multicolumn{6}{|c|}{$0 \leq|y|<0.5, \sqrt{S}=13 \mathrm{TeV}$} \\
\hline$K_{0.2}$ & 0.75 & 0.77 & 0.81 & 0.83 & 0.85 \\
\hline$K_{0.4}$ & 0.85 & 0.87 & 0.88 & 0.91 & 0.91 \\
\hline$K_{0.7}$ & 0.94 & 0.95 & 0.96 & 0.97 & 0.97 \\
\hline$K_{0.9}$ & 0.98 & 0.98 & 0.99 & 1.01 & 1.01 \\
\hline
\end{tabular}

of the NLO + NLL and the NLO cross sections for different jet radii as a function of the signal-jet $p_{T}$ and $|y|<0.5$ at both $\sqrt{S}=7 \mathrm{TeV}$ (left) and $13 \mathrm{TeV}$ (right). Results for selected values of $p_{T}$ are listed in Table I. For all numerical calculations in this section we use the MMHT14
PDFs [64] at NLO as an example. We find that for a large range of the jet $p_{T}$, the joint resummation leads to a decrease of the NLO cross section. The effect is more pronounced for smaller values of $R$, where the impact of the $\ln (R)$ resummation becomes more noticeable and leads to a significant decrease of the cross section. For larger values of the jet $p_{T}$, the threshold enhancement will compensate the $\ln (R)$ contributions and can eventually result in an enhancement. This effect is most clearly illustrated in Fig. 1 and Table I for $R=0.9$ for $p_{T}$ around $\mathcal{O}(1 \mathrm{TeV})$. A similar trend has been observed in recent work on threshold resummation with a parton shower event generator including quantum interference [65].

To illustrate the dependence of the cross section on the jet radius parameter $R$ we plot in Figs. 2 and 3 for both, fixed-order NLO and NLO + NLL resummed predictions, the respective ratios $D_{R}$

$$
D_{R}=\frac{\sigma(R)}{\sigma\left(R_{\text {fixed }}\right)},
$$

as a function of the signal-jet $p_{T}$ and $|y|<0.5$ for the energies $\sqrt{S}=7$ and $13 \mathrm{TeV}$. Nonperturbative (NP) correction factors are taken from [17] and results for selected
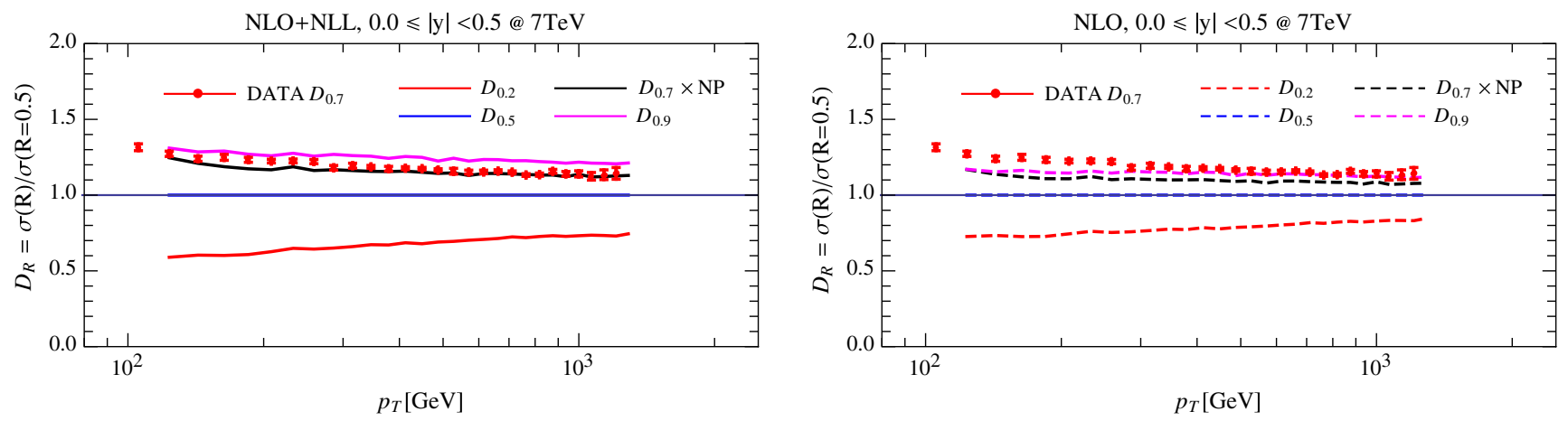

FIG. 2. Ratios $D_{R}$ of Eq. (5) with $R_{\text {fixed }}=0.5$ at $\sqrt{S}=7 \mathrm{TeV}$ for the cross sections at NLO + NLL (left) and NLO (right) accuracy using the MMHT PDF set [64] at NLO with NP correction factors which are taken from [17]. The red dots indicate the single-inclusive jet data for $D_{R}$ from CMS collected at $\sqrt{S}=7 \mathrm{TeV}$ with $R=0.7$ [17].
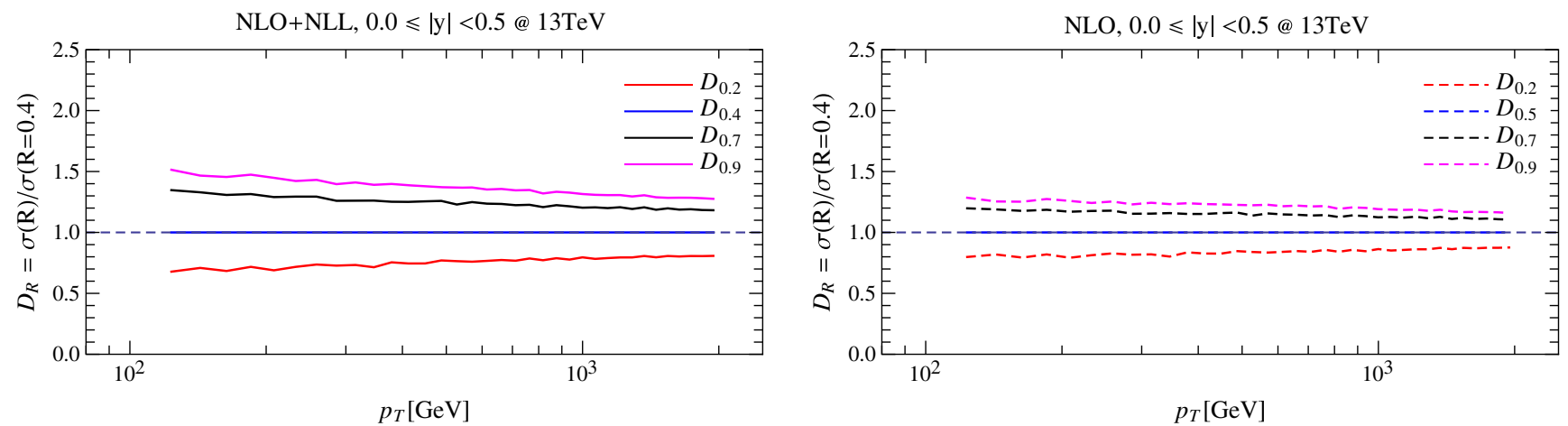

FIG. 3. Ratios $D_{R}$ of Eq. (5) with $R_{\text {fixed }}=0.4$ at $\sqrt{S}=13 \mathrm{TeV}$ for the cross sections at NLO + NLL (left) and NLO (right) accuracy using the MMHT PDF set [64] at NLO. 
TABLE II. Ratios of Eq. (5) for the cross sections at NLO and $\mathrm{NLO}+\mathrm{NLL}$ accuracy denoted by $D_{R}^{\mathrm{NLO}}$ and $D_{R}^{\mathrm{NLO}+\mathrm{NLL}}$, respectively, at $\sqrt{S}=7 \mathrm{TeV}$ (top) and $13 \mathrm{TeV}$ (bottom) for selected values of the signal jet $p_{T}$ using the MMHT PDF set [64] at NLO. The results at $\sqrt{S}=7 \mathrm{TeV}$ include NP correction factors which are taken from [17].

\begin{tabular}{|c|c|c|c|c|c|}
\hline$p_{T}[\mathrm{GeV}]$ & \multicolumn{2}{|c|}{200} & 300 & 500 & 1000 \\
\hline \multicolumn{6}{|c|}{$0 \leq|y|<0.5, \sqrt{S}=7 \mathrm{TeV}$} \\
\hline$D_{0.2}^{\mathrm{NLO}+\mathrm{NLL}}$ & \multicolumn{2}{|c|}{0.62} & 0.65 & 0.69 & 0.73 \\
\hline$D_{0.5}^{\mathrm{NLO}+\mathrm{NLL}}$ & \multicolumn{2}{|c|}{1.00} & 1.00 & 1.00 & 1.00 \\
\hline$D_{0.7}^{\mathrm{NLO}+\mathrm{NLL}}$ & \multicolumn{2}{|c|}{1.16} & 1.16 & 1.14 & 1.12 \\
\hline$D_{0.9}^{\mathrm{NLO}+\mathrm{NLL}}$ & \multicolumn{2}{|c|}{1.27} & 1.26 & 1.22 & 1.21 \\
\hline$D_{0.2}^{\mathrm{NLO}}$ & \multicolumn{2}{|c|}{0.73} & 0.76 & 0.78 & 0.82 \\
\hline$D_{0.5}^{\mathrm{NLO}}$ & \multicolumn{2}{|c|}{1.00} & 1.00 & 1.00 & 1.00 \\
\hline$D_{0.7}^{\mathrm{NLO}}$ & \multicolumn{2}{|c|}{1.10} & 1.09 & 1.08 & 1.07 \\
\hline$D_{0.9}^{\mathrm{NLO}}$ & \multicolumn{2}{|c|}{1.14} & 1.14 & 1.13 & 1.11 \\
\hline$p_{T}[\mathrm{GeV}]$ & 200 & 300 & 500 & 1000 & 1500 \\
\hline \multicolumn{6}{|c|}{$0 \leq|y|<0.5, \sqrt{S}=13 \mathrm{TeV}$} \\
\hline$D_{0.2}^{\mathrm{NLO}+\mathrm{NLL}}$ & 0.70 & 0.74 & 0.76 & 0.79 & 0.80 \\
\hline$D_{0.4}^{\mathrm{NLO}+\mathrm{NLL}}$ & 1.00 & 1.00 & 1.00 & 1.00 & 1.00 \\
\hline$D_{0.7}^{\mathrm{NLO}+\mathrm{NLL}}$ & 1.30 & 1.27 & 1.24 & 1.21 & 1.19 \\
\hline$D_{0.9}^{\mathrm{NLO}+\mathrm{NLL}}$ & 1.46 & 1.40 & 1.37 & 1.31 & 1.29 \\
\hline$D_{0.2}^{\mathrm{NLO}}$ & 0.80 & 0.81 & 0.84 & 0.86 & 0.86 \\
\hline$D_{0.4}^{\mathrm{NLO}}$ & 1.00 & 1.00 & 1.00 & 1.00 & 1.00 \\
\hline$D_{0.7}^{\mathrm{NLO}}$ & 1.17 & 1.15 & 1.15 & 1.12 & 1.11 \\
\hline$D_{0.9}^{\mathrm{NLO}}$ & 1.26 & 1.23 & 1.22 & 1.19 & 1.16 \\
\hline
\end{tabular}

values of $p_{T}$ are listed in Table II. The predicted dependence of the cross section on the jet radius parameter $R$ tends to be more pronounced when the effects of the joint resummation are considered. For the smaller value of $R=0.2$, the ratio with resummation $D_{R}^{\mathrm{NLO}+\mathrm{NLL}}$ is smaller

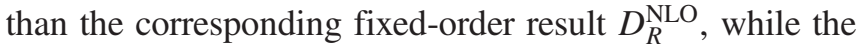
opposite trend is observed for larger radii $R \geq 0.7$, cf. Table II. We emphasize that the ratios $D_{R}$ are quite insensitive to the chosen input PDFs. This leads to precise

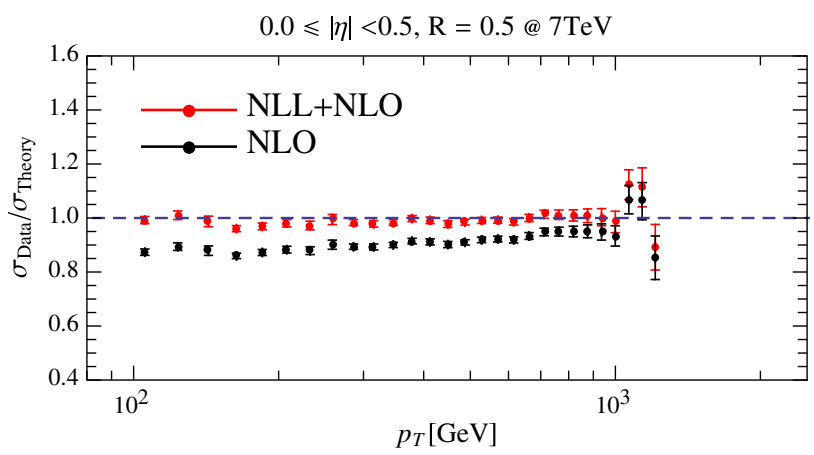

predictions of the NLL + NLO and the NLO calculations which are experimentally well testable. In Fig. 2, we have performed a comparison of predictions with the inclusive jet data for $D_{0.7}$ shown in red dots measured by CMS [17] at the LHC with at $\sqrt{S}=7 \mathrm{TeV}$. The error bars represent the experimental uncertainties which are small due to the cancellation of systematic errors in the ratio. After considering the NP effects, the NLO + NLL resummed result in Fig. 2 (left) agrees very well with the measurement while the fixed-order NLO prediction in Fig. 2 (right) fails to describe the data for the entire range of jet $p_{T}$ considered. We note that those CMS data for $D_{0.7}$ are even larger than the fixed-order NLO prediction $D_{0.9}$, i.e., a situation which intuitively should be reversed.

As a further illustration of the resummation effects we compare the predicted central values at fixed NLO and at $\mathrm{NLO}+\mathrm{NLL}$ accuracy in Eq. (3) with the LHC data [17,22], collected at $\sqrt{S}=7$ and $13 \mathrm{TeV}$, respectively. In Figs. 4 and 5, the LHC data in the rapidity bin $|y|<0.5$ are normalized to the theoretical predictions and displayed as a function of the signal-jet $p_{T}$. For $\sqrt{S}=7 \mathrm{TeV}$ the NP effects have been included in the predictions in Fig. 4. It is clearly visible how the resummed predictions lead to an increase of the ratio of cross sections $\sigma_{\text {Data }} / \sigma_{\text {Theory }}$ compared to the NLO result for all choices of jet radii, so that the NLO + NLL results of Eq. (3) are in perfect agreement with the $\sqrt{S}=7 \mathrm{TeV}$ data [17]. For the $\sqrt{S}=13 \mathrm{TeV}$ data [22] with the choice of $R=0.4$, the resummation improves the theory description as well, whereas the data for $R=0.7$ slightly overshoots the theory predictions. However, the experimental uncertainties of those data sets are still relatively large.

The observations presented here do neither depend significantly on the scales chosen as the leading jet transverse momentum $\mu_{F}=\mu_{R}=p_{T}^{\max }$ nor on the PDFs. This will be quantified in detail in the following sections.

\section{B. Scale dependence}

In this section, we analyze in detail the scale dependence of the jointly resummed single-inclusive jet cross section.

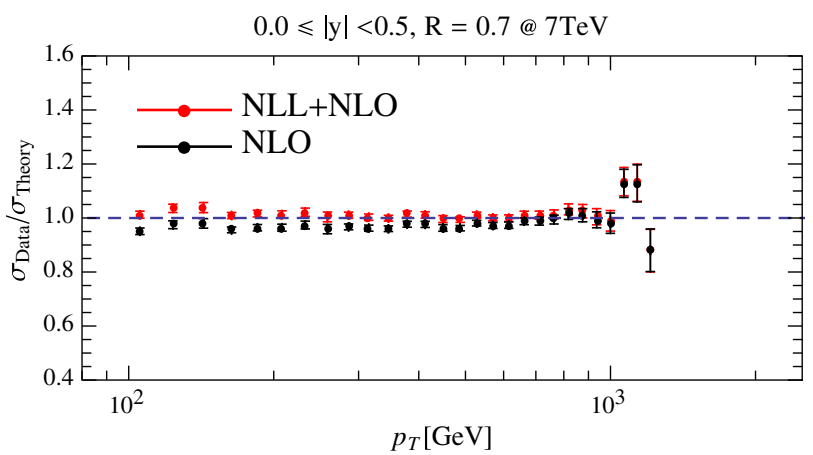

FIG. 4. The ratio $\sigma_{\text {Data }} / \sigma_{\text {Theory }}$ for the CMS data $\sqrt{S}=7 \mathrm{TeV}$ of [17] with $R=0.5$ (left) and $R=0.7$ (right) to the theoretical results at NLO (black) and at NLO + NLL (red) accuracy using the MMHT PDF set [64] at NLO. 

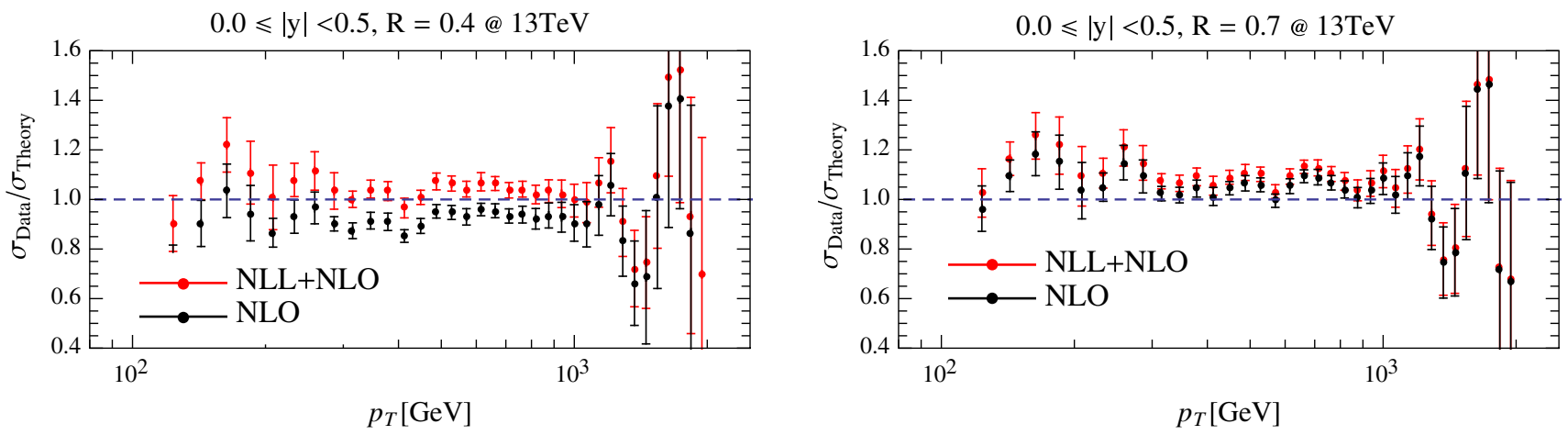

FIG. 5. Same as Fig. 4 for the CMS data of [22] at $\sqrt{S}=13 \mathrm{TeV}$ with $R=0.4$ (left) and $R=0.7$ (right).

It is instructive to compare the obtained scale dependence to the case where only the logarithms in the jet size parameter $\ln (R)$ are resummed [30]. In Fig. 6, we show the residual scale uncertainty of the jointly resummed cross section normalized to NLO. The scale band is obtained as discussed in Sec. II above. In addition, we show the cross section where only $\ln (R)$ terms are resummed. In this case, the scale band is obtained by varying only the hard scale $\mu_{h}$ and the jet scale $\mu_{J}$ by factors of two around their canonical choices. One observes a significant reduction of the residual scale dependence once also threshold resummation is taken into account. This observation holds true even though for the small- $R$ resummed calculation there are only two scales that are varied in order to estimate the QCD uncertainty whereas there are three separate scales that are all varied independently for the jointly resummed result. One also notices that the reduction of the scale uncertainty gets more pronounced at higher jet transverse momenta where threshold resummation is more relevant. In fact, this behavior is generally expected for threshold resummed calculations and has been analyzed in more detail before in many instances, see for example the studies for Higgs boson hadro-production $[66,67]$. When approaching large $p_{T}$, the joint resummation surpasses the small- $R$ resummed cross section due to the threshold enhancement.

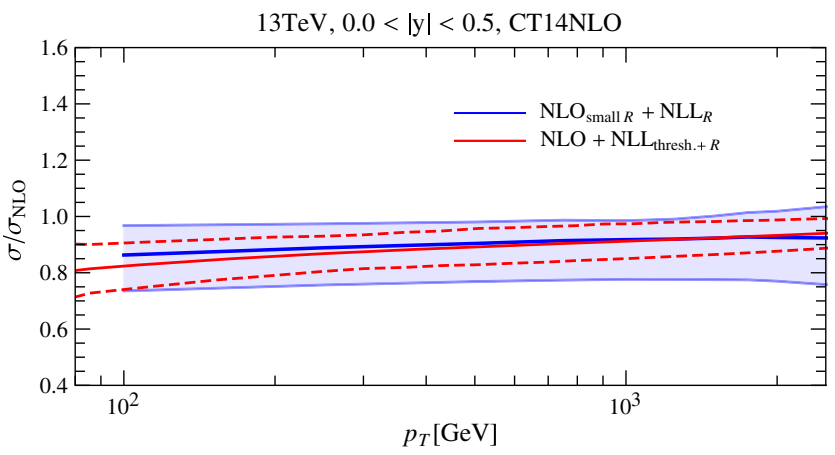

FIG. 6. Comparison of the scale dependence of the jointly resummed cross section (red) and the case where only small- $R$ logarithms are resummed to all orders (blue) [30]. As an example, we use the CT14 PDF set [68] at NLO with $R=0.4$ and both results are normalized to NLO. See text for more details.
An important caveat here is that the $\ln (R)$ resummed calculation of [30] can currently be performed only with the scale choice $\mu=p_{T}$ whereas in the threshold limit we always have $\mu=p_{T}^{\max }$. This difference is most relevant at small values of the jet transverse momentum and likely explains the difference of the central values of the two curves at small $p_{T}$. On the other hand, it is interesting to note that for both scale choices, the resummation consistently leads to a suppression relative to the respective NLO calculation. In addition, the jointly resummed calculation is matched and normalized to the full NLO. Instead, the $\ln (R)$ resummed calculation is using the narrow jet approximation. However, the differences are of order $\mathcal{O}\left(R^{2}\right)$ which are negligible for $R=0.4$ [69,70].

\section{Comparison to LHC data}

Now we move on to the comparison of the theoretical predictions with the CMS inclusive jet analyses for both $\sqrt{S}=7$ and $13 \mathrm{TeV}[17,22]$. Other data sets, such as those by CMS collected at $\sqrt{S}=2.76 \mathrm{TeV}$ [15] and the one ATLAS at $\sqrt{S}=13 \mathrm{TeV}$ [21] have already been considered in [27].

We start with $\sqrt{S}=7 \mathrm{TeV}$ following the CMS analysis [17] and focus on the cross section data with the anti- $k_{T}$ jet radius $R=0.5$, which we bin into four different rapidity regions: $0.0 \leq|y|<0.5,0.5 \leq|y|<1.0,1.0 \leq|y|<1.5$ and $1.5 \leq|y|<2.0$. For each rapidity bin, we present the pQCD predictions $\sigma_{\mathrm{NLO}+\mathrm{NLL}}$ at NLO $+\mathrm{NLL}$ and $\sigma_{\mathrm{NLO}}$ at NLO accuracy based on the CT10 PDFs [71] at NLO as in the original CMS analysis [17].

Figures 7 and 8 show the ratio of the CMS data to the theoretical predictions, that is $\sigma_{\text {Data }} / \sigma_{\text {Theory }}$ for both NLO + NLL and NLO accuracy. For both cases, the NP effects as provided by CMS [17] have been included in the perturbative calculations to convert the predictions from the parton level to the particle level. The yellow bands in Figs. 7 and 8 indicate the theoretical uncertainties from scale variations obtained as discussed in the previous section with the hard scale chosen as $\mu=p_{T}^{\max }$. The solid brown lines on the other hand indicate the experimental 

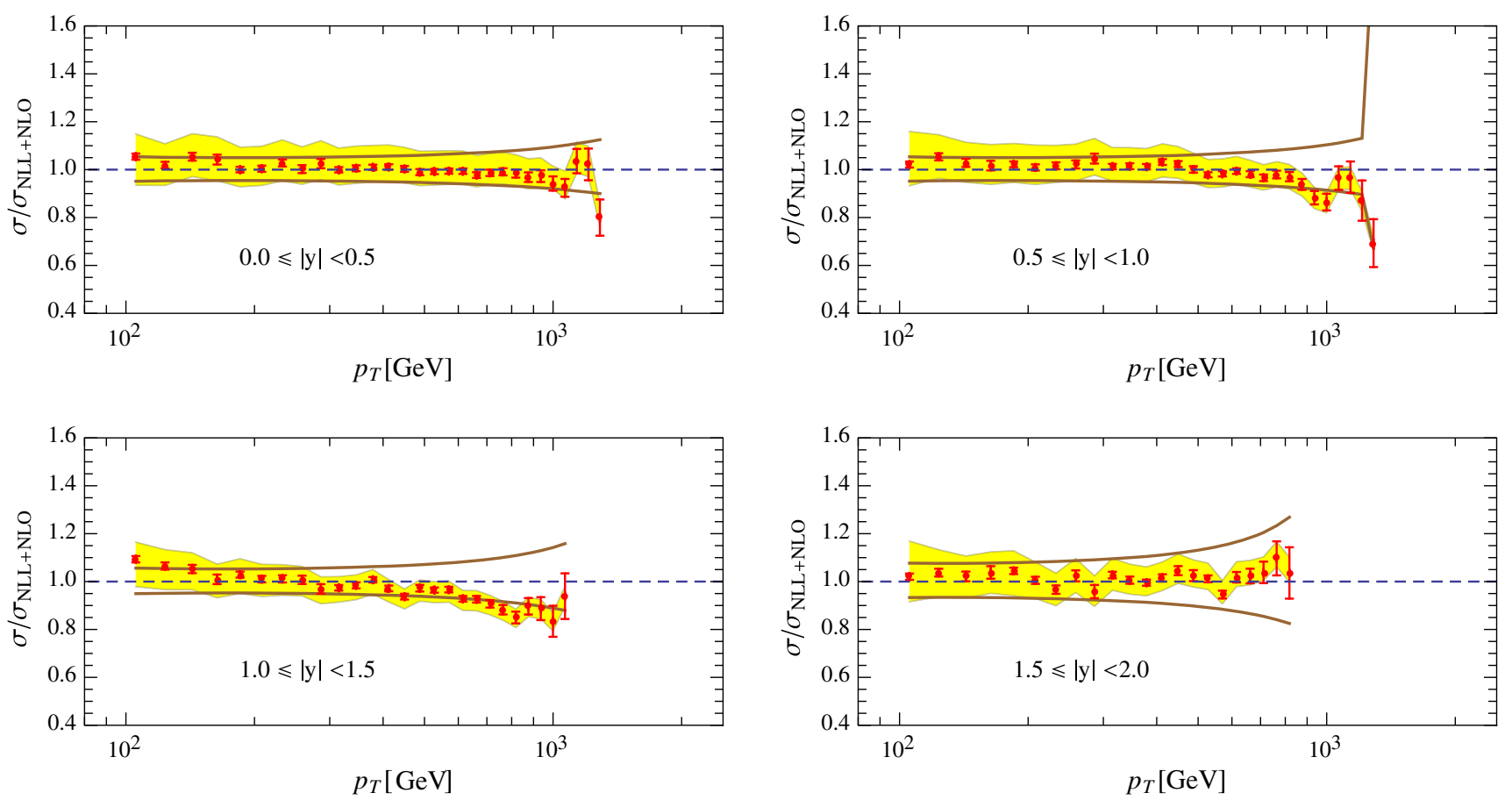

FIG. 7. The ratio $\sigma_{\text {Data }} / \sigma_{\mathrm{NLO}+\mathrm{NLL}}$ for the CMS data collected at $\sqrt{S}=7 \mathrm{TeV}$ [17] with $R=0.5$ to the theoretical results using the CT10 PDF set [71] at NLO. The error bars represent the experimental statistical errors and the solid (brown) lines the systematic ones. The band (yellow) indicates theoretical scale uncertainties. The NP corrections from [17] have been included.

systematic errors, whereas the error bars on the data represent the experimental statistical errors [17].

In Fig. 7, we observe very good agreement with the data in all rapidity regions for the NLO + NLL predictions
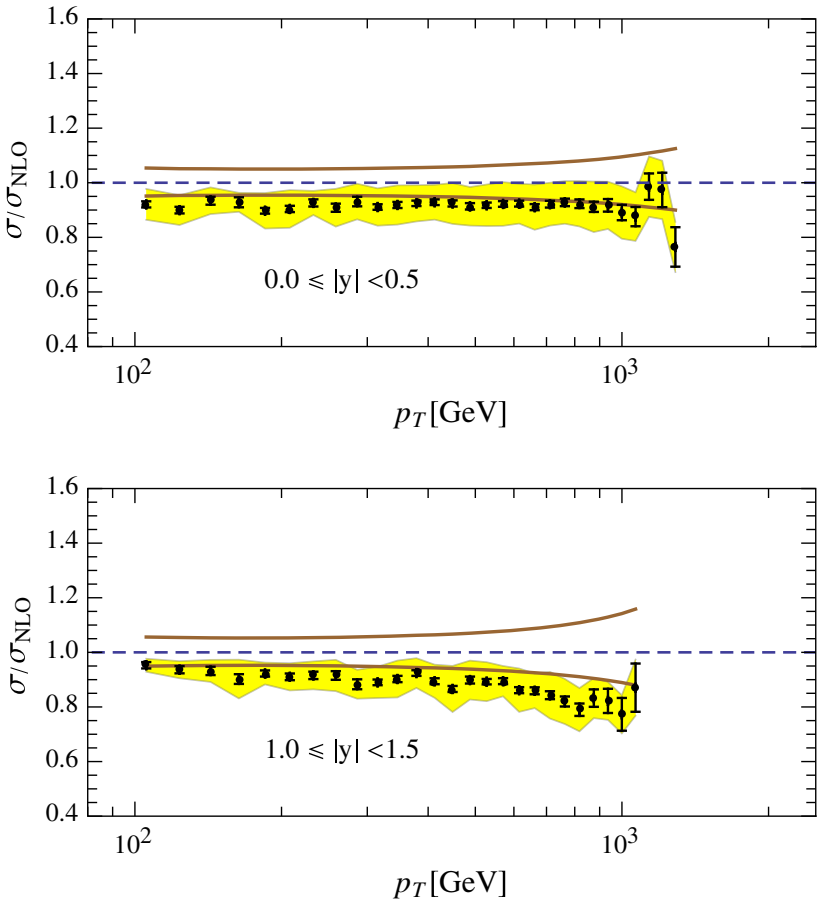

where the NLL joint resummation is taken into account. In the high- $p_{T}$ region, the NLO + NLL calculations still somewhat overestimate the CMS data. However, this can be further improved by switching from the CT10 PDFs [71]
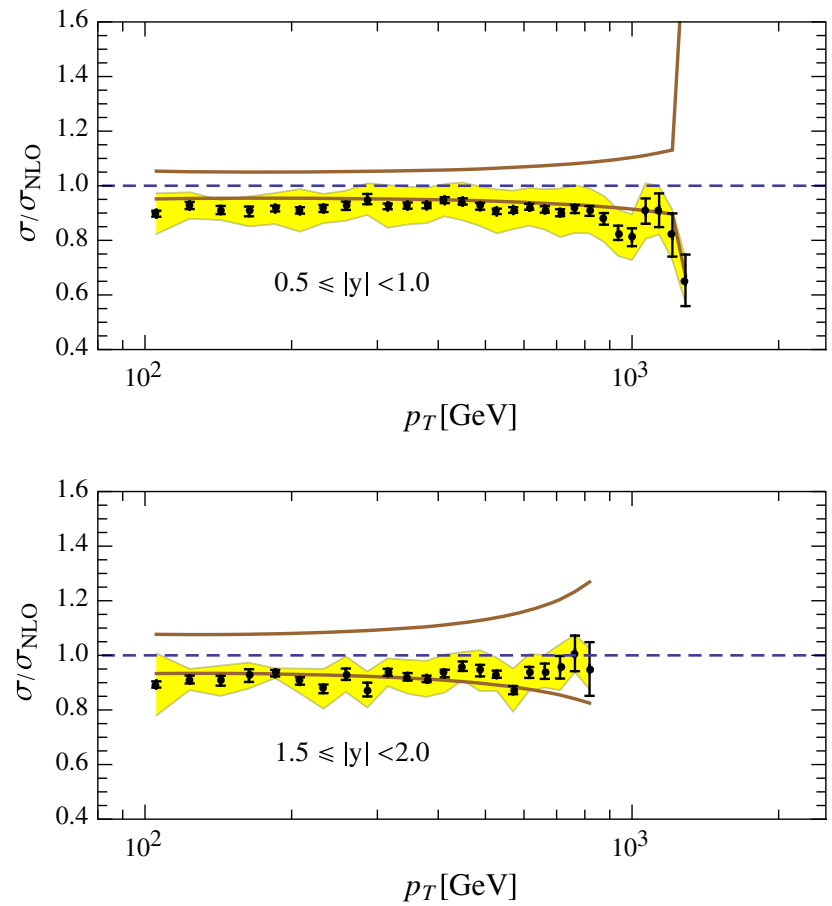

FIG. 8. Same as Fig. 7 for the ratio $\sigma_{\text {Data }} / \sigma_{\mathrm{NLO}}$. 
to more recent PDF sets, as we will detail in the next section. In contrast, the NLO results in Fig. 8 are consistently larger than the inclusive jet data by an amount of $10 \%$ in all rapidity bins. Thus, all predictions are lying along the lower boundary of the systematic errors (brown lines) in Fig. 8, except for the high $p_{T}$ tail region of the rapidity bin $1.5 \leq|y|<2.0$.

The theoretical uncertainty of the NLL + NLO predictions in Fig. 7 is still large and comparable with the experimental errors. However, this can be reduced further in the future with the help improved accuracy for the resummation, i.e., upon resumming the relevant logarithms to NNLL accuracy together with matching to the available NNLO calculations.

Next we study the inclusive jet production cross section with $R=0.4$ at $\sqrt{S}=13 \mathrm{TeV}$. The results are shown in Figs. 9 and 10, in which the ratio of the CMS data [22] to the cross sections $\sigma_{\mathrm{NLO}+\mathrm{NLL}}$ and $\sigma_{\mathrm{NLO}}$ are displayed, respectively. Here, we have applied the CT14 PDF set [68] at NLO for both predictions and we note that the NP and the electroweak effects have not been included in this analysis. Again, the yellow band in Figs. 9 and 10 represents the theoretical scale uncertainties whereas the experimental systematic and statistical errors are shown as solid brown lines and the error bars, respectively. At present, the published CMS data at $\sqrt{S}=13 \mathrm{TeV}$ have larger statistical errors, since they are based on data samples corresponding to a relatively small integrated luminosities of 71 and 44 inverse picobarns, whereas the $\sqrt{S}=7 \mathrm{TeV}$ data [17] discussed above correspond to an integrated luminosity of 5.0 inverse femtobarns.

As shown in Fig. 9, the NLO + NLL calculation leads to a good agreement of the ratio $\sigma_{\text {Data }} / \sigma_{\text {Theory }}$ with unity in the region of central rapidities, but slightly overshoots it in the rapidity bin $1.5 \leq|y|<2.0$, although still being compatible within the errors. On the contrary, in Fig. 10 the ratio $\sigma_{\text {Data }} / \sigma_{\text {Theory }}$ based on the NLO predictions systematically undershoots unity in the rapidity region $|y|<1.5$, but it is still compatible within the quoted uncertainties. Better consistency of the NLO results with the CMS data is only observed in the rapidity region $1.5 \leq|y|<2.0$.

\section{Impact of different PDFs}

We now proceed to study the sensitivity of different choices of PDFs in predicting the inclusive jet $p_{T}$ distributions. We benchmark our study using the CMS data at $\sqrt{S}=7 \mathrm{TeV}$ since the experimental errors of those data are relatively small. Besides the CT10 PDFs used above, we also consider the following alternative PDF extractions which are available in the literature to NLO and NNLO accuracy in pQCD: ABMP16 [72,73], CT14 [68], HERAPDF2.0 [74], MMHT2014 [64] and NNPDF3.1 [75]. In addition, we use the PDF set of [39] obtained within the framework of NNPDF by fitting only data for the Drell-Yan (DY) process, deep-inelastic scattering (DIS) and
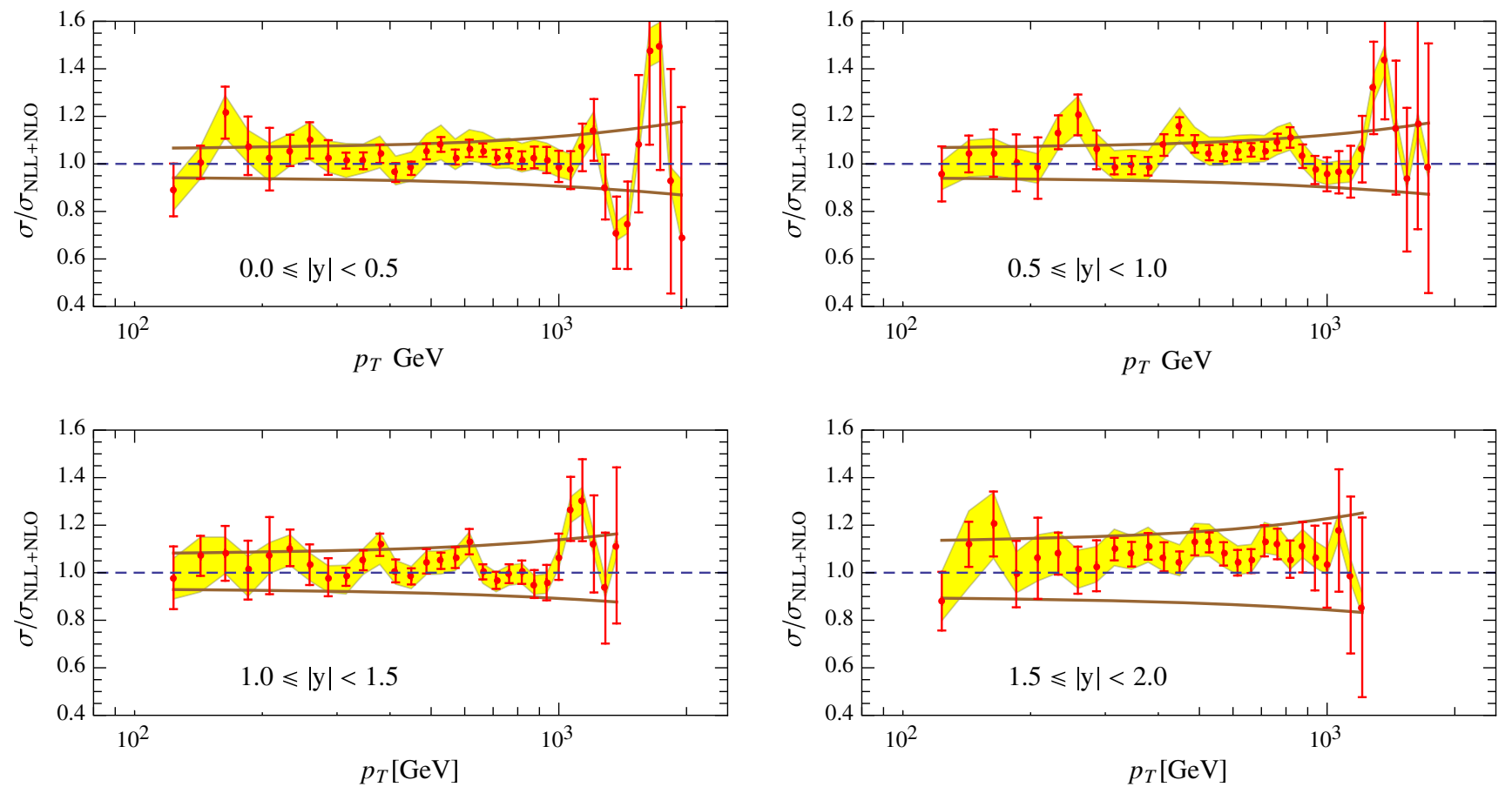

FIG. 9. The ratio $\sigma_{\text {Data }} / \sigma_{\mathrm{NLO}+\mathrm{NLL}}$ for CMS data collected at $\sqrt{S}=13 \mathrm{TeV}$ [22] with $R=0.4$ to the theoretical results using the PDFs of CT14 [68] at NLO. The error bars represent the experimental statistical errors and the solid (brown) lines the systematic ones. The band (yellow) indicates theoretical scale uncertainties. 

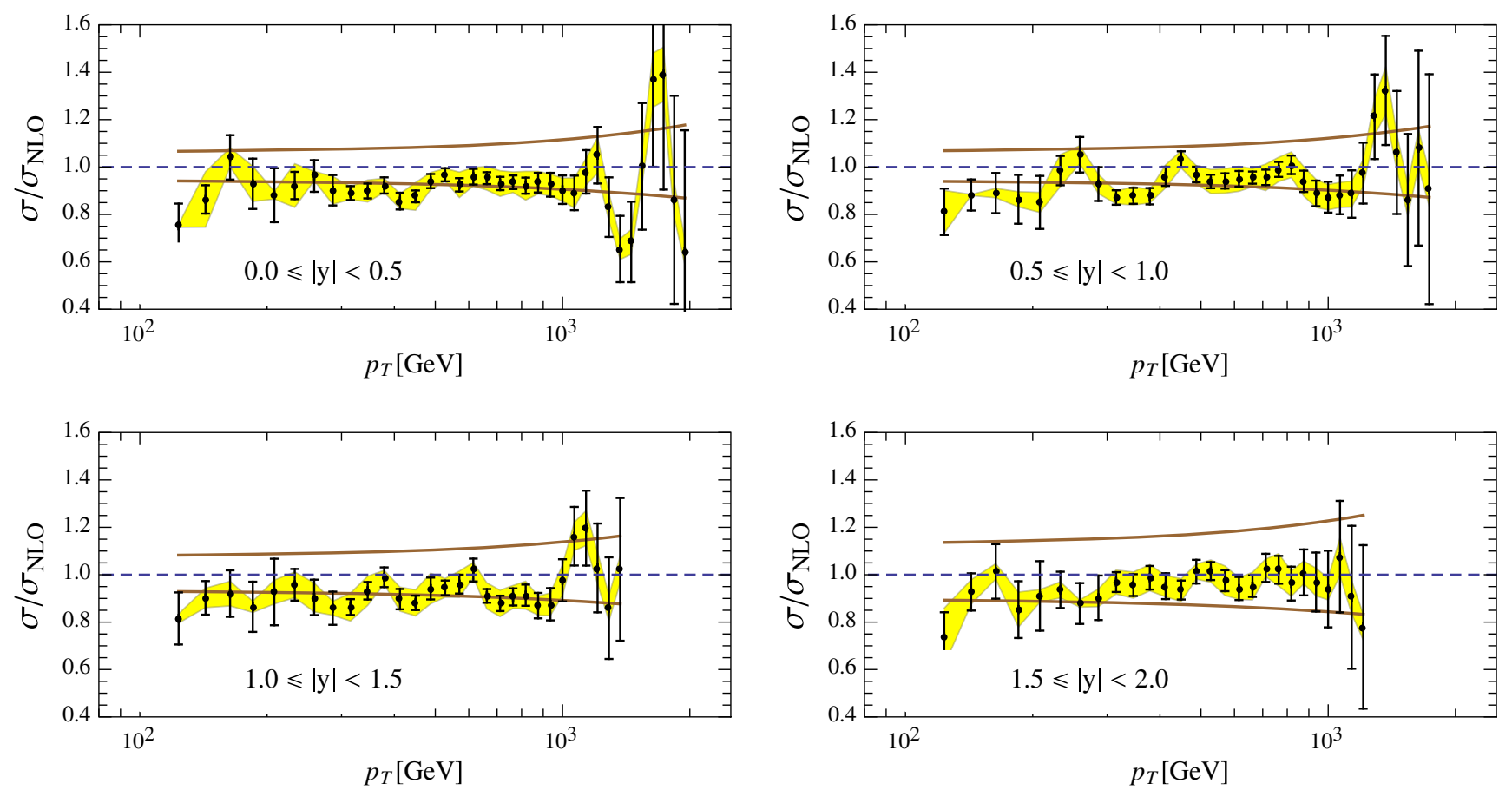

FIG. 10. Same as Fig. 9 for the ratio $\sigma_{\text {Data }} / \sigma_{\mathrm{NLO}}$.

top-quark hadro-production but including threshold resummation in all theory predictions for the hard scattering.

The NLO + NLL studies are presented in Figs. 11 and 12, and the NLO ones in Figs. 13 and 14, respectively. To
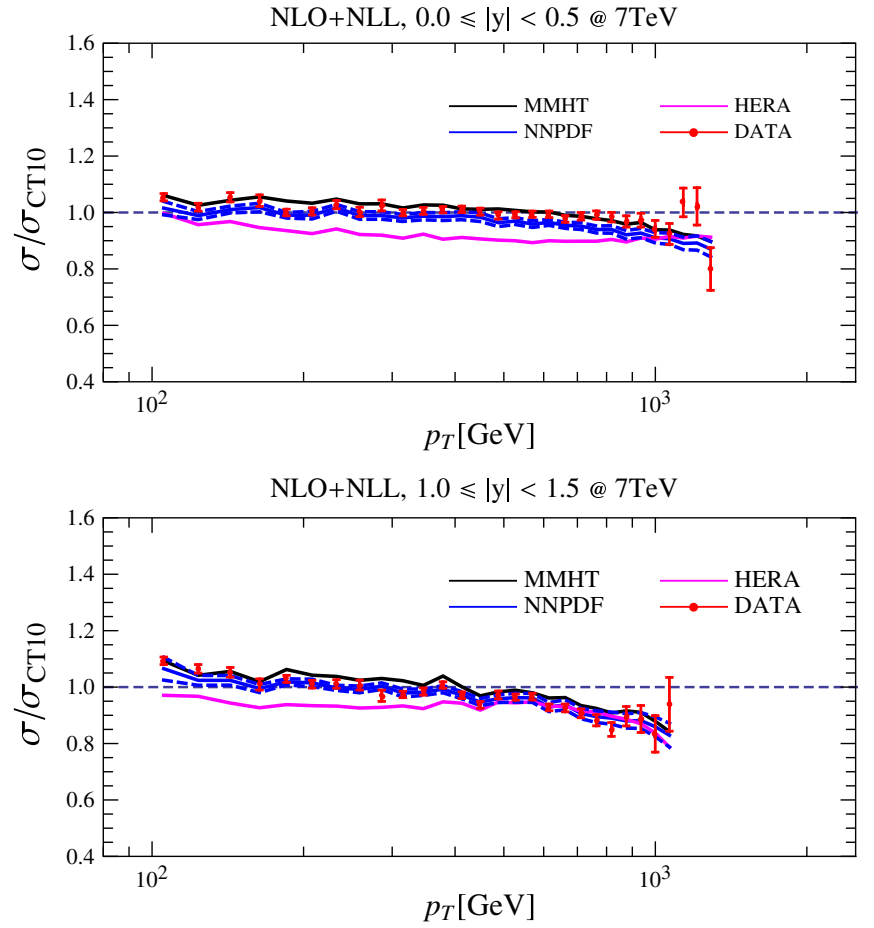

maintain consistency, the NLO variants for all PDFs are used here, the value of the strong coupling $\alpha_{s}\left(M_{Z}\right)$ is taken as provided by the respective PDF sets and the predictions are normalized to the one with CT10 PDFs at NLO to allow

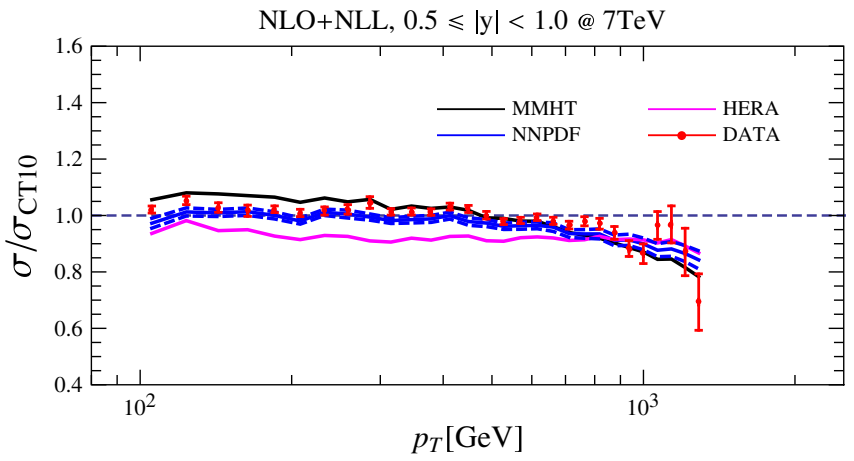

NLO+NLL, $1.5 \leqslant|y|<2.0 @ 7 \mathrm{TeV}$

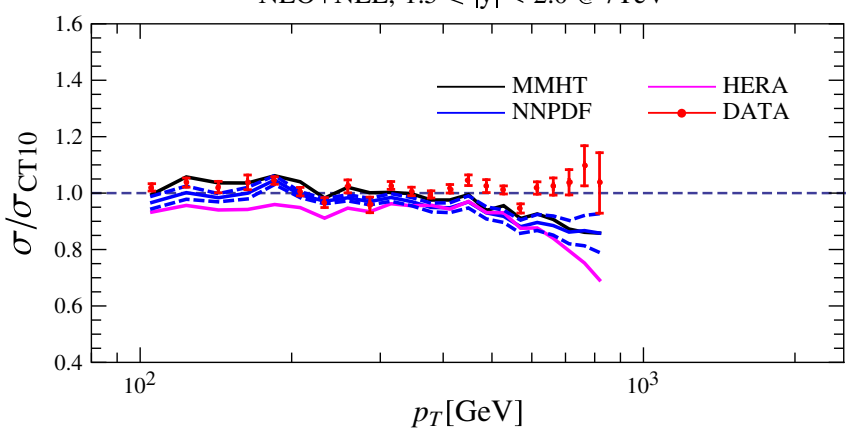

FIG. 11. Cross sections $\sigma_{\mathrm{NLO}+\mathrm{NLL}}$ at NLL + NLO accuracy with $R=0.5$ using the central PDF sets HERAPDF2.0 [74], MMHT2014 [64] and NNPDF3.1 [75] normalized to the one with CT10 PDFs [71] at NLO. The dashed lines (blue) indicate the PDF uncertainties for the NNPDF3.1 set. The CMS data collected at $\sqrt{S}=7 \mathrm{TeV}[17]$ with $R=0.5$ with their experimental statistical errors are displayed as dots (red). 

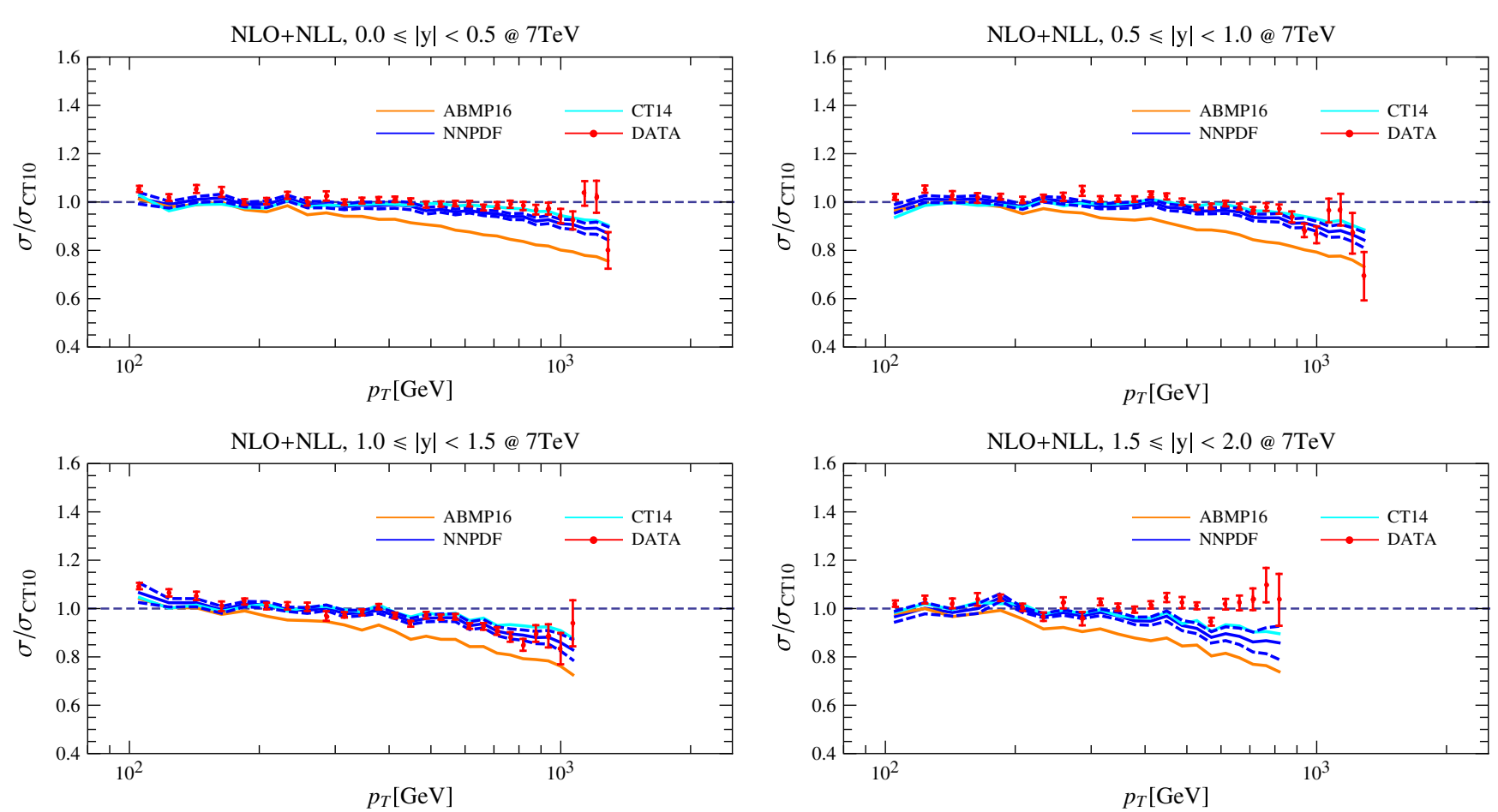

FIG. 12. Same as Fig. 11 for the PDF sets ABMP16 [72], CT14 [68] and NNPDF3.1 [75].

for comparisons with the CMS analysis [17]. In Figs. 11 and 12 , we see that the predictions based on the NLL joint resummation achieve excellent agreement with the experimental data for the PDF sets CT14, MMHT2014 and NNPDF3.1, while the PDF set ABMP16 slightly undershoots the data for large values of $p_{T}$. Only in the
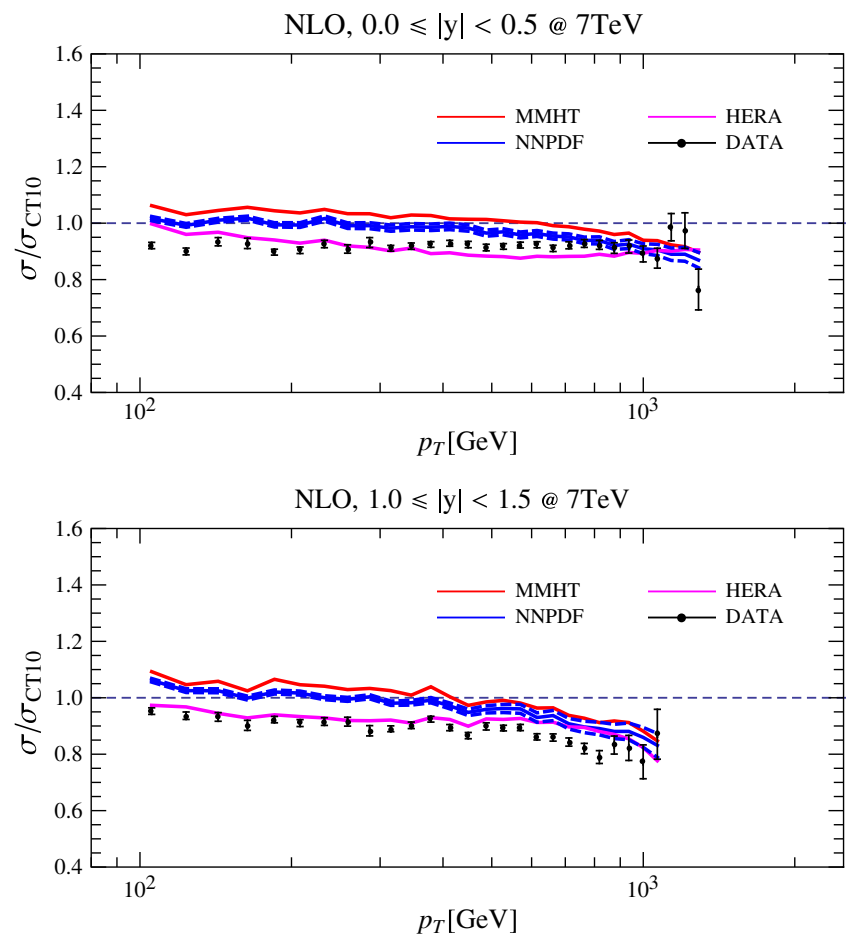

high- $p_{T}$ region of the bin with rapidities $1.5 \leq|y|<2.0$, the data are in excess of the theoretical predictions, but the discrepancy is still within the experimental error, see Fig. 7. The predictions with HERAPDF2.0 show a somewhat different trend. They are lower than the data in most of the $p_{T}$ region and only tend to agree with the data in the
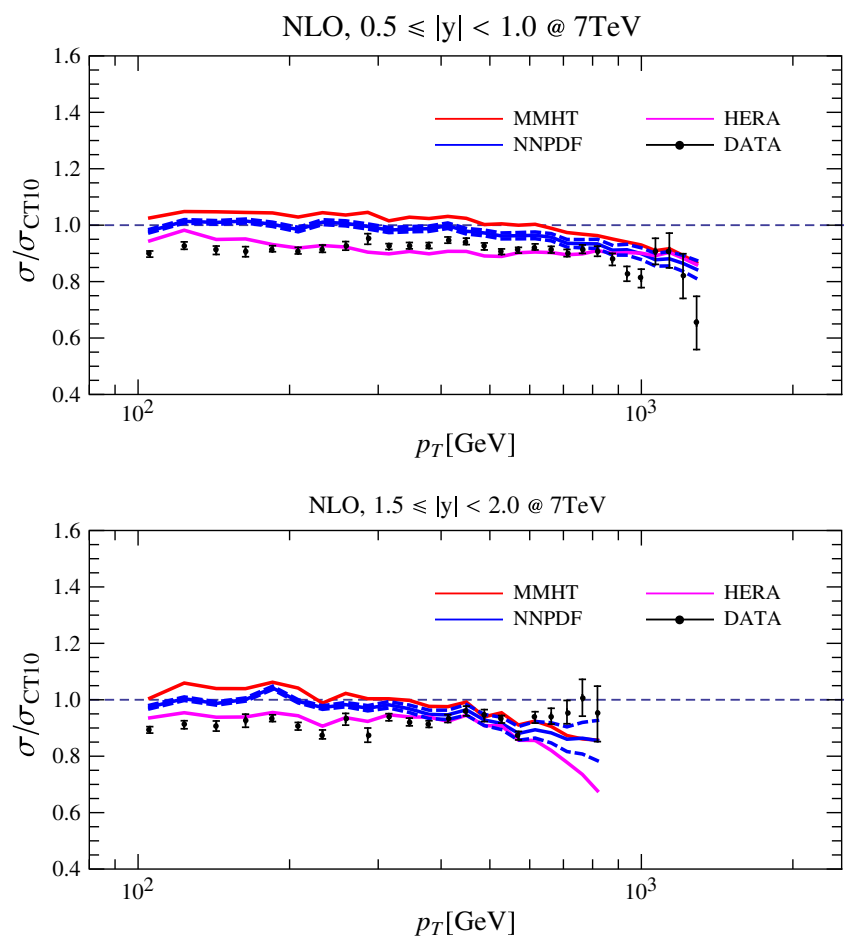

FIG. 13. Same as Fig. 11 for cross sections $\sigma_{\mathrm{NLO}}$ at NLO in pQCD. 

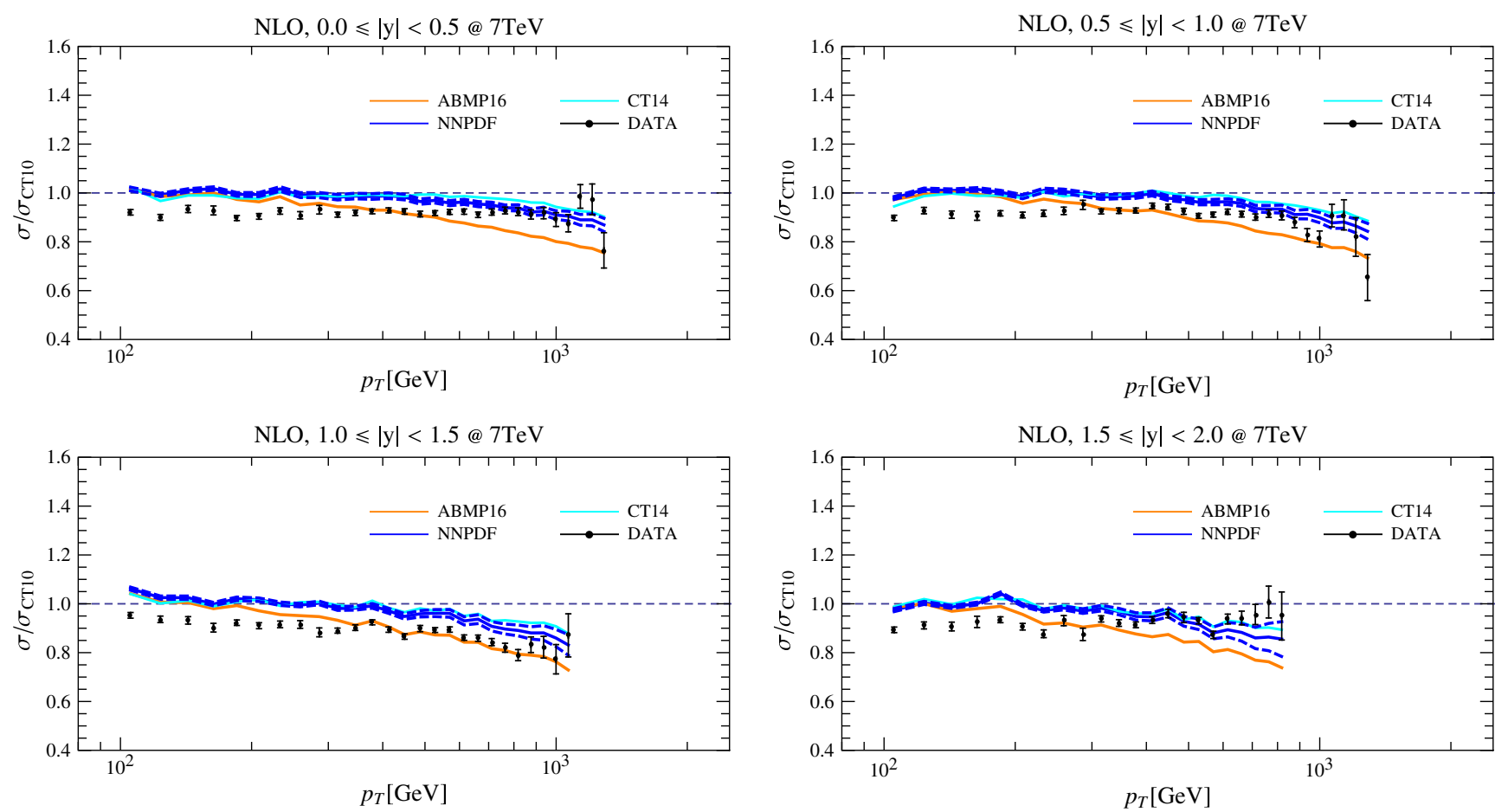

FIG. 14. Same as Fig. 12 for cross sections $\sigma_{\mathrm{NLO}}$ at NLO in pQCD.

high- $p_{T}$ regime around $p_{T} \simeq 1 \mathrm{TeV}$. To illustrate the robustness of the observations further, we also plot the uncertainties for the NNPDF3.1 PDFs, which turn out to be quite small compared with the theoretical error from the scale uncertainty, see again Fig. 7. The uncertainties for other PDFs are of similar size.

The situation deteriorates substantially when only the NLO corrections are taken into account. For $\sigma_{\mathrm{NLO}}$ at NLO, as shown in Figs. 13 and 14, none of the PDFs do a good job in describing the CMS inclusive jet data. The predictions with ABMP16, CT14, MMHT2014 and NNPDF3.1 are all higher than the data in all rapidity bins in most of the jet $p_{T}$ regions. For $p_{T} \gtrsim 800 \mathrm{GeV}$ those predictions tend to agree with or slightly undershoot the data for rapidities $|y|<1.5$. For HERAPDF2.0 the NLO predictions are within the experimental errors of the inclusive jet data, but the shape of the $p_{T}$ dependence of the cross section differs slightly from the one of the data. Like in the NLO + NLL case above, we also display the uncertainties for the NNPDF3.1 PDF sets in Figs. 13 and 14. As the PDF errors are found to be small, the theory predictions are rather stable against the uncertainties of current PDFs from global fits and the strong tension between the NLO theory and the data persists. We also note, that recent studies [24,25] found it to be impossible to re-constrain the PDFs within a global analysis including inclusive jet data from the LHC when all current crosscorrelations among different rapidity bins are consistently taken into account.

Figure 15 shows again the comparison of the NLO + NLL calculations with the CMS data at $\sqrt{S}=7 \mathrm{TeV}$ but now using the NNLO variants of the PDF sets under study. This choice is reasonable to a certain extent, since the NLL resummation includes a dominant part of the full NNLO contributions. On the other hand, potentially large NNLO corrections, for instance possible large corrections from the complete two-loop virtual corrections are still missing in $\sigma_{\mathrm{NLO}+\mathrm{NLL}}$. We can see from Fig. 15 that the NNLO variants of CT14, MMHT2014, NNPDF3.1 get slightly shifted, but are well consistent with the CMS data, again except for the highest values of $p_{T}$ in the rapidity bin $1.5 \leq|y|<2$.0. In contrast, the predictions with the NNLO variant of HERAPDF2.0 are significantly shifted compared to the NLO one, cf. Fig. 11, and display now also good consistency with the CMS data. The NNLO variant of the AMBP16 PDFs predicts the correct shape, but it is lower than the data for all rapidity bins as a consequence of the lower value of $\alpha_{s}\left(M_{Z}\right)=0.1147$ compared to $\alpha_{s}\left(M_{Z}\right)=$ 0.1180 used by CT14, HERAPDF2.0, MMHT2014 or NNPDF3.1. This sensitivity to $\alpha_{s}\left(M_{Z}\right)$ confirms again the great potential of inclusive jet cross section data for the determination of the strong coupling constant [23].

Finally, in Fig. 16, we display the results with the PDFs of [39] which have been extracted from data for the DY process, DIS and top-quark hadro-production within the NNPDF framework. These PDFs are subject to improvements at large- $x$, since the theory predictions for DIS and DY as well as for top-quark hadro-production include threshold resummation. For the PDF variant without threshold resummation (labeled as NNPDF30NLO in Fig. 16) both cross sections at NLO + NLL and NLO accuracy, $\sigma_{\mathrm{NLO}+\mathrm{NLL}}$ and $\sigma_{\mathrm{NLO}}$, respectively, are shown. In 

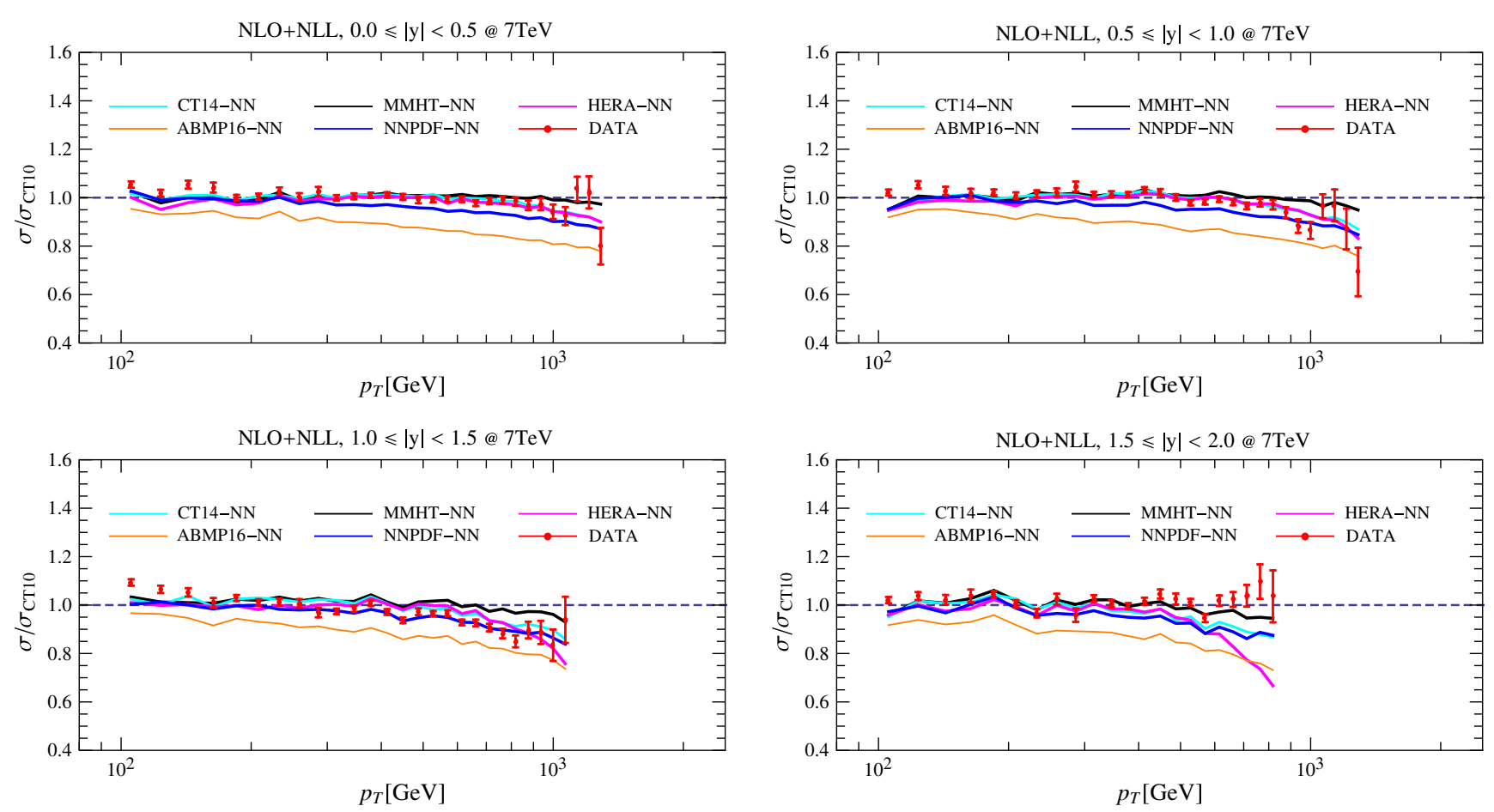

FIG. 15. Same as Fig. 11 with the NNLO variant taken for all PDF sets ABMP16 [73], CT14 [68], HERAPDF2.0 [74], MMHT2014 [64] and NNPDF3.1 [75].

addition to that, the NLO + NLL results $\sigma_{\mathrm{NLO}+\mathrm{NLL}}$ for the PDF variant with threshold resummation (labeled as NNPDF 30 NLL in Fig. 16) are presented as well. Overall, the NLO + NLL predictions exhibit better agreement with
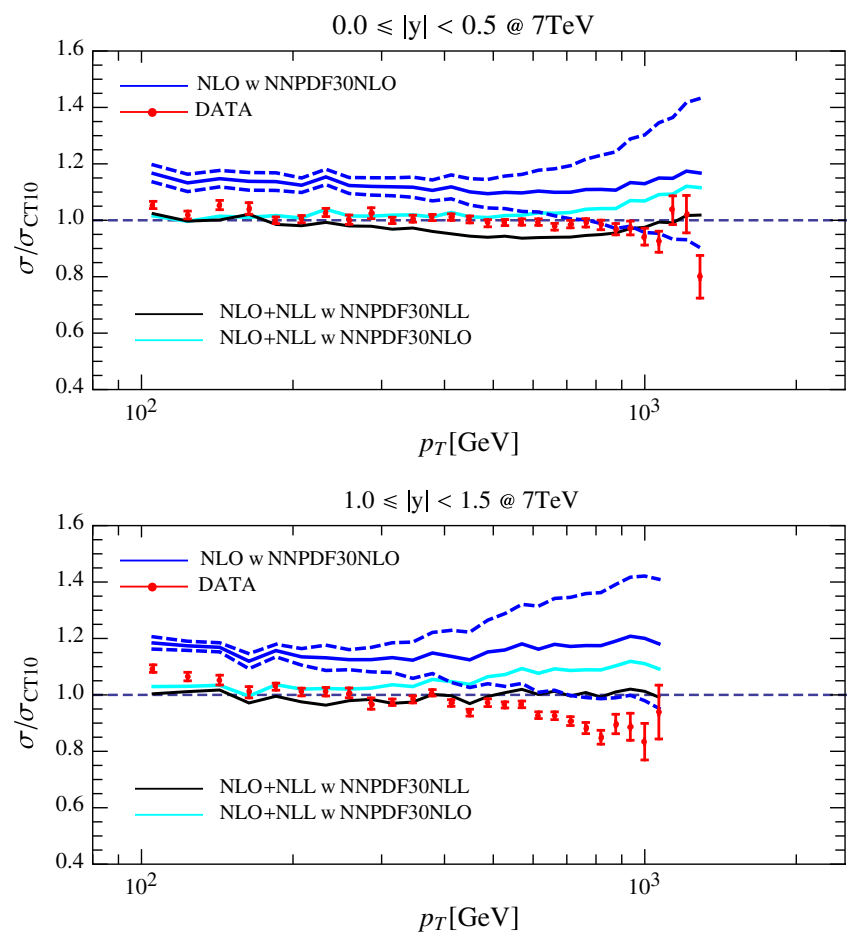

the data compared to the NLO results, although the PDFs uncertainties of [39] are substantially larger than the ones of global fits. Those large PDF uncertainties at large- $x$ and relevant scales of $p_{T} \simeq \mu \simeq 0.5 \ldots 1 \mathrm{TeV}$ originate from the
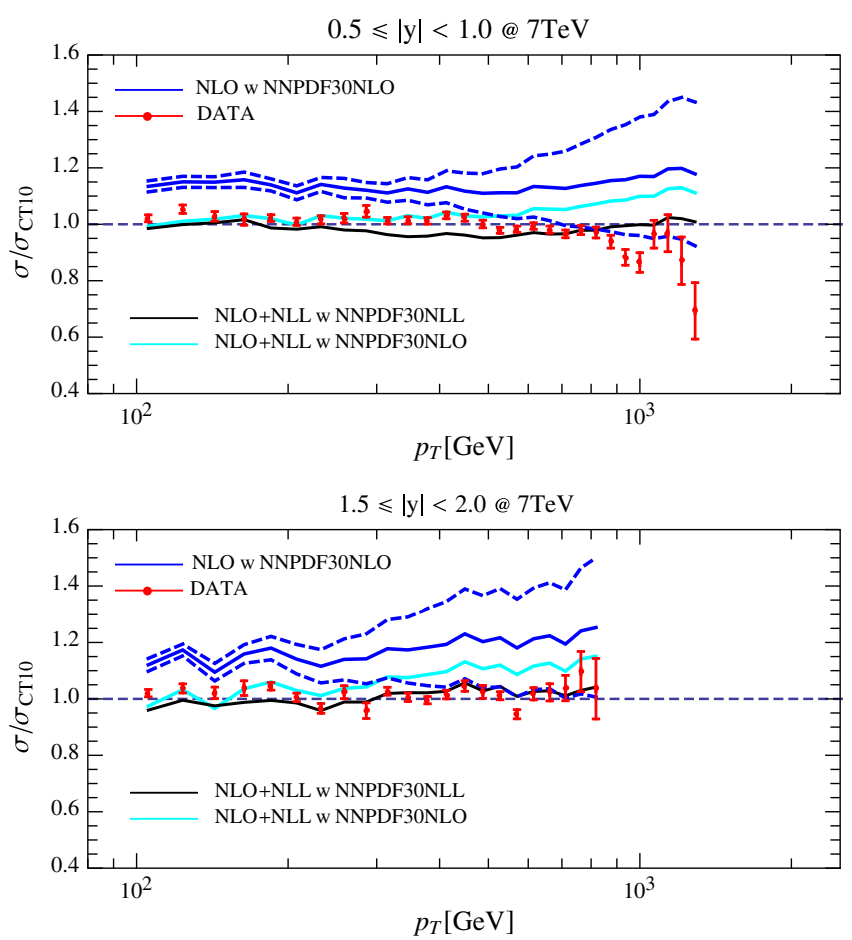

FIG. 16. Same as in Figs. 11 and 13 for the cross sections $\sigma_{\text {NLO+NLL }}$ and $\sigma_{\text {NLO }}$ using the PDF sets NNPDF3.0 at NLO without and with resummation [39], labeled as NNPDF30NLO and NNPDF3 ONLL in the plots, respectively. The dashed lines (blue) indicate the PDF uncertainties for $\sigma_{\mathrm{NLO}}$ with the set NNPDF30NLO. 
gluon PDF at $x \gtrsim 0.1$ and the light flavor PDFs at lower $x$ through the standard parton evolution. The findings in Fig. 16 underpin the necessity to carefully examine and analyze data which constrain those PDFs, including the need to delineate resummation effects from power corrections in the kinematic regions. It will be interesting to observe to what extent improvements can be made in the future in extractions of PDFs with threshold resummation when the inclusive jet data are included.

\section{SUMMARY AND CONCLUSIONS}

In this manuscript, we have provided a detailed study of pQCD calculations from first principles for cross sections of single-inclusive jet production at the LHC. We have performed comprehensive comparisons between the fixed NLO results and the NLL threshold and small- $R$ joint resummation improved calculations obtained recently, and we have achieved remarkable advances in perturbative predictions upon using the latter. In our studies, significant differences between the NLO and the NLO + NLL joint resummation predictions have been observed in the kinematic regions of interest for the LHC analyses and we have found that these differences account for the discrepancy between the NLO predictions and the LHC data for the jet $p_{T}$ spectrum in various rapidity bins collected by the CMS experiment at various center-ofmass energies. Once the joint resummation has been included, a remarkable agreement was found between the QCD theory predictions and the LHC data in a large range of jet rapidities.

We have illustrated the impact of the joint resummation in a study of the jet radius ratios $D_{R}$ at both $\sqrt{S}=7$ and $13 \mathrm{TeV}$, which have the advantage of being largely independent of the PDFs and other residual theory uncertainties. At $\sqrt{S}=7 \mathrm{TeV}$ these jet radius ratios between $R=0.5$ and $R=0.7$, i.e., $D_{0.7}$, have been compared with a CMS analysis in Fig. 2 and overall we have found a significant improvement in the theoretical description of those data. While the predicted double-differential cross sections in $p_{T}$ and $y$ at NLO in $\mathrm{pQCD}$ are, for a given value of $R$, systematically higher than the central values of those LHC data in all rapidity bins, arguably they still agree within the theoretical and experimental uncertainties. For the jet radius ratios $D_{R}$, however, such consistency is definitely not the case due to the much reduced experimental uncertainties. The NLO predictions for $D_{0.7} \mathrm{com}$ pletely miss those LHC data and also cannot be changed by considering different PDF sets since those effects largely cancel out in the jet radius ratios $D_{R}$. Therefore, we conclude that the NLO + NLL joint resummation is a crucial ingredient in order to achieve a good description of the $\sqrt{S}=7 \mathrm{TeV}$ jet data within pQCD. We have also presented predictions for the jet radius ratios $D_{R}$ at $\sqrt{S}=13 \mathrm{TeV}$ in Fig. 3 using different jet radii with a jet $p_{T}$ up to $2 \mathrm{TeV}$. These results will be useful for future experimental analyses of inclusive jet data.

Due to the great importance of the inclusive jet data for constraints on PDFs and determinations of the strong coupling $\alpha_{s}\left(M_{Z}\right)$ we have also investigated in this study the impact of different PDF sets on the theoretical predictions. We have found that the NLO + NLL predictions at $\sqrt{S}=7 \mathrm{TeV}$ based on the NLO variants of the PDF sets ABMP16, CT14, MMHT2014 and NNPDF3.1 or on the NNLO variant of HERAPDF2.0, respectively, describe the $p_{T}$ distributions remarkably well for the various rapidity bins. On the other hand, the tension with the CMS inclusive jet data and the pure NLO predictions $\sigma_{\mathrm{NLO}}$ persists for all those PDF sets and cannot be removed or relieved by selecting a specific PDF set. Therefore, global PDFs which also fit inclusive jet data from the LHC need to be based on theory predictions using the joint resummation for the single-inclusive jet production in order to avoid a possible bias in the PDF extraction due to missing large logarithms in the hard cross sections beyond NLO. We have also noticed in our studies that PDFs extracted with account of threshold resummation but without inclusive jet data have significantly increased PDF uncertainties compared to the ones from the global fits. We suggest to use the joint resummed calculations of the present article in the ongoing efforts to refine those PDFs.

Finally, we note that although the NLO + NLL calculations greatly improve the theoretical predictions, the associated scale uncertainties are still large and comparable with the current experimental errors. Therefore, in future studies it will be necessary to go beyond the currently achieved accuracy by matching the resummed results with the now available inclusive jet calculations at NNLO and by improving the logarithmic accuracy of the joint threshold and small- $R$ joint resummation to NNLL. Both these tasks are feasible within the present framework for resummation and will be subject of future work.

\section{ACKNOWLEDGMENTS}

We would like to thank Jun Gao and Katerina Lipka for helpful discussions and Marco Bonvini for providing the PDF set of [39]. X. L. would like to thank Zhong-Bo Kang and the UCLA Department of Physics and Astronomy and Deutsches Elektronen-Synchrotron DESY for the hospitality. X.L. is supported by the National Natural Science Foundation of China under Grant No. 11775023 and the Fundamental Research Funds for the Central Universities. S.-O. M. acknowledges Contract No. 05H15GUCC1 by BMBF. F. R. is supported by the U.S. Department of Energy (DOE) under Contract No. DE-AC02-05CH11231 and by the Laboratory Directed Research and Development (LDRD) Program of Lawrence Berkeley National Laboratory. This research used resources of the Argonne Leadership Computing Facility, which is a DOE Office of Science User Facility supported under Contract No. DEAC02-06CH11357. 


\section{APPENDIX: CROSS SECTIONS AT $\sqrt{S}=13 \mathrm{TeV}$}

We present the cross sections for the LHC in Tables III-VI, respectively, including the theory uncertainty arising from scale variations. At NLO, the scale uncertainties have been obtained from the envelope of the variation around $\mu_{R}=\mu_{F}=p_{T}^{\max }$ up

TABLE III. The double-differential cross sections $\sigma_{\mathrm{NLO}+\mathrm{NLL}}$ in bins of $p_{T}$ and $y$ at $\sqrt{S}=13 \mathrm{TeV}$ with $R=0.4$ using the CT14 [68] PDFs at NLO. Theoretical uncertainties from the scale variation are given in parenthesis.

\begin{tabular}{|c|c|c|c|c|c|c|}
\hline \multicolumn{2}{|c|}{$p_{T}[\mathrm{GeV}]$} & \multicolumn{5}{|c|}{$d \sigma / d p_{T} / d|y|[\mathrm{pb}]\left( \pm \Delta \sigma_{\text {th scale }}\right.$ in $\left.\%\right)$} \\
\hline$p_{T}^{\min }$ & $p_{T}^{\max }$ & $0 \leq|y|<0.5$ & $0.5 \leq|y|<1.0$ & $1.0 \leq|y|<1.5$ & $1.5 \leq|y|<2.0$ & $2.0 \leq|y|<2.5$ \\
\hline \multicolumn{7}{|c|}{$\sigma_{\mathrm{NLO}+\mathrm{NLL}}$ for LHC at $\sqrt{S}=13 \mathrm{TeV}, R=0.4$} \\
\hline 56 & 74 & $1.20 \times 10^{+5}( \pm 15.06 \%)$ & $1.17 \times 10^{+5}( \pm 13.72 \%)$ & $1.07 \times 10^{+5}( \pm 14.10 \%)$ & $8.99 \times 10^{+4}( \pm 19.41 \%)$ & $7.25 \times 10^{+4}( \pm 25.95 \%)$ \\
\hline 74 & 97 & $3.49 \times 10^{+4}( \pm 10.61 \%)$ & $3.28 \times 10^{+4}( \pm 11.83 \%)$ & $2.95 \times 10^{+4}( \pm 12.10 \%)$ & $2.47 \times 10^{+4}( \pm 15.22 \%)$ & $1.93 \times 10^{+4}( \pm 21.42 \%)$ \\
\hline 97 & 133 & $8.76 \times 10^{+3}( \pm 9.53 \%)$ & $8.23 \times 10^{+3}( \pm 10.41 \%)$ & $7.31 \times 10^{+3}( \pm 10.91 \%)$ & $6.04 \times 10^{+3}( \pm 12.44 \%)$ & $4.71 \times 10^{+3}( \pm 17.60 \%)$ \\
\hline 133 & 174 & $2.05 \times 10^{+3}( \pm 8.50 \%)$ & $1.93 \times 10^{+3}( \pm 8.34 \%)$ & $1.69 \times 10^{+3}( \pm 8.24 \%)$ & $1.37 \times 10^{+3}( \pm 9.75 \%)$ & $1.02 \times 10^{+3}( \pm 15.06 \%)$ \\
\hline 174 & 220 & $5.66 \times 10^{+2}( \pm 7.96 \%)$ & $5.27 \times 10^{+2}( \pm 8.06 \%)$ & $4.55 \times 10^{+2}( \pm 8.41 \%)$ & $3.63 \times 10^{+2}( \pm 8.38 \%)$ & $2.60 \times 10^{+2}( \pm 13.37 \%)$ \\
\hline 220 & 272 & $1.75 \times 10^{+2}( \pm 7.24 \%)$ & $1.63 \times 10^{+2}( \pm 7.04 \%)$ & $1.39 \times 10^{+2}( \pm 7.10 \%)$ & $1.08 \times 10^{+2}( \pm 7.46 \%)$ & $7.26 \times 10^{+1}( \pm 12.22 \%)$ \\
\hline 272 & 330 & $5.91 \times 10^{+1}( \pm 6.86 \%)$ & $5.42 \times 10^{+1}( \pm 6.60 \%)$ & $4.57 \times 10^{+1}( \pm 6.22 \%)$ & $3.43 \times 10^{+1}( \pm 7.08 \%)$ & $2.14 \times 10^{+1}( \pm 11.40 \%)$ \\
\hline 330 & 395 & $2.11 \times 10^{+1}( \pm 7.07 \%)$ & $1.93 \times 10^{+1}( \pm 6.25 \%)$ & $1.59 \times 10^{+1}( \pm 5.80 \%)$ & $1.15 \times 10^{+1}( \pm 6.65 \%)$ & $6.42 \times 10^{+0}( \pm 10.81 \%)$ \\
\hline 395 & 468 & $7.91 \times 10^{+0}( \pm 6.93 \%)$ & $7.16 \times 10^{+0}( \pm 6.10 \%)$ & $5.79 \times 10^{+0}( \pm 5.18 \%)$ & $3.95 \times 10^{+0}( \pm 6.48 \%)$ & $1.90 \times 10^{+0}( \pm 10.41 \%)$ \\
\hline 468 & 548 & $3.07 \times 10^{+0}( \pm 7.22 \%)$ & $2.75 \times 10^{+0}( \pm 6.00 \%)$ & $2.17 \times 10^{+0}( \pm 4.45 \%)$ & $1.37 \times 10^{+0}( \pm 6.35 \%)$ & $5.44 \times 10^{-1}( \pm 10.00 \%)$ \\
\hline 548 & 638 & $1.23 \times 10^{+0}( \pm 7.03 \%)$ & $1.09 \times 10^{+0}( \pm 5.96 \%)$ & $8.28 \times 10^{-1}( \pm 3.99 \%)$ & $4.78 \times 10^{-1}( \pm 6.29 \%)$ & $1.48 \times 10^{-1}( \pm 9.61 \%)$ \\
\hline 638 & 737 & $4.98 \times 10^{-1}( \pm 7.02 \%)$ & $4.36 \times 10^{-1}( \pm 5.73 \%)$ & $3.17 \times 10^{-1}( \pm 3.51 \%)$ & $1.62 \times 10^{-1}( \pm 6.31 \%)$ & $3.67 \times 10^{-2}( \pm 9.15 \%)$ \\
\hline 737 & 846 & $2.07 \times 10^{-1}( \pm 6.86 \%)$ & $1.77 \times 10^{-1}( \pm 5.49 \%)$ & $1.22 \times 10^{-1}( \pm 3.35 \%)$ & $5.32 \times 10^{-2}( \pm 6.33 \%)$ & $8.05 \times 10^{-3}( \pm 8.77 \%)$ \\
\hline 846 & 967 & $8.63 \times 10^{-2}( \pm 6.93 \%)$ & $7.23 \times 10^{-2}( \pm 5.12 \%)$ & $4.61 \times 10^{-2}( \pm 3.45 \%)$ & $1.66 \times 10^{-2}( \pm 6.34 \%)$ & $1.50 \times 10^{-3}( \pm 8.41 \%)$ \\
\hline 967 & 1101 & $3.58 \times 10^{-2}( \pm 6.73 \%)$ & $2.92 \times 10^{-2}( \pm 4.81 \%)$ & $1.70 \times 10^{-2}( \pm 3$ & $4.79 \times 10^{-3}( \pm 6$ & $2.19 \times 10^{-4}( \pm 8.04 \%)$ \\
\hline 1101 & 1248 & $1.49 \times 10^{-2}( \pm 6.69 \%)$ & $1.17 \times 10^{-2}( \pm 4.58 \%)$ & $6.05 \times 10^{-3}( \pm 3.84 \%)$ & $1.25 \times 10^{-3}( \pm 6.39 \%)$ & $2.21 \times 10^{-5}( \pm 8.57 \%)$ \\
\hline 1248 & 1410 & $6.09 \times 10^{-3}( \pm 6.53 \%)$ & $4.57 \times 10^{-3}( \pm 4.23 \%)$ & $2.05 \times 10^{-3}( \pm 4.03 \%)$ & $2.86 \times 10^{-4}( \pm 6.42 \%)$ & $1.18 \times 10^{-6}( \pm 9.93 \%)$ \\
\hline 1410 & 1588 & $2.45 \times 10^{-3}( \pm 6.35 \%)$ & $1.73 \times 10^{-3}( \pm 3.84 \%)$ & $6.49 \times 10^{-4}( \pm 4.22 \%)$ & $5.50 \times 10^{-5}( \pm 6.48 \%)$ & $1.75 \times 10^{-8}( \pm 12.79 \%)$ \\
\hline 1588 & 1784 & $9.60 \times 10^{-4}( \pm 6.23 \%)$ & $6.30 \times 10^{-4}( \pm 3.50 \%)$ & $1.88 \times 10^{-4}( \pm 4.41 \%)$ & $8.30 \times 10^{-6}( \pm 6.55 \%)$ & $5.45 \times 10^{-12}( \pm 23.09 \%)$ \\
\hline 1784 & 2000 & $3.60 \times 10^{-4}( \pm 6.03 \%)$ & $2.16 \times 10^{-4}( \pm 3.16 \%)$ & $4.85 \times 10^{-5}( \pm 4.61 \%)$ & $8.57 \times 10^{-7}( \pm 6.64 \%)$ & $0.45 \times 10$ \\
\hline 2000 & 2238 & $1.28 \times 10^{-4}( \pm 5.89 \%)$ & $6.84 \times 10^{-5}( \pm 2.86 \%)$ & $1.07 \times 10^{-5}( \pm 4.81 \%)$ & $4.69 \times 10^{-8}( \pm 7.07 \%)$ & . \\
\hline 2238 & 2500 & $4.25 \times 10^{-5}( \pm 5.70 \%)$ & $1.96 \times 10^{-5}( \pm 2.59 \%)$ & $1.91 \times 10^{-6}( \pm 5.02 \%)$ & $7.58 \times 10^{-10}( \pm 8.68 \%)$ & - \\
\hline 2500 & 2787 & $1.29 \times 10^{-5}( \pm 5.49 \%)$ & $4.91 \times 10^{-6}( \pm 2.79 \%)$ & $2.57 \times 10^{-7}( \pm 5.23 \%)$ & $4.71 \times 10^{-13}( \pm 12.81 \%)$ & - \\
\hline 2787 & 3103 & $3.51 \times 10^{-6}( \pm 5.22 \%)$ & $1.04 \times 10^{-6}( \pm 3.03 \%)$ & $2.25 \times 10^{-8}( \pm 5.47 \%)$ & & - \\
\hline
\end{tabular}

TABLE IV. Same as Table III for the cross sections $\sigma_{\mathrm{NLO}}$.

\begin{tabular}{|c|c|c|c|c|c|c|}
\hline \multicolumn{2}{|c|}{$p_{T}[\mathrm{GeV}]$} & \multicolumn{5}{|c|}{$d \sigma / d p_{T} / d|y|[\mathrm{pb}]\left( \pm \Delta \sigma_{\text {th scale }}\right.$ in $\left.\%\right)$} \\
\hline$\overline{p_{T}^{\min }}$ & $p_{T}^{\max }$ & $0 \leq|y|<0.5$ & $0.5 \leq|y|<1.0$ & $1.0 \leq|y|<1.5$ & $1.5 \leq|y|<2.0$ & $2.0 \leq|y|<2.5$ \\
\hline \multicolumn{7}{|c|}{$\sigma_{\mathrm{NLO}}$ for LHC at $\sqrt{S}=13 \mathrm{TeV}, R=0.4$} \\
\hline 56 & 74 & $1.52 \times 10^{+5}( \pm 2.60 \%)$ & $1.48 \times 10^{+5}( \pm 3.96 \%)$ & $1.36 \times 10^{+5}( \pm 5.17 \%)$ & $1.17 \times 10^{+5}( \pm 4.59 \%)$ & $9.57 \times 10^{+4}( \pm 7.17 \%)$ \\
\hline 74 & 97 & $4.28 \times 10^{+4}( \pm 6.03 \%)$ & $4.05 \times 10^{+4}( \pm 3.83 \%)$ & $3.66 \times 10^{+4}( \pm 5.55 \%)$ & $3.11 \times 10^{+4}( \pm 5.34 \%)$ & $2.47 \times 10^{+4}( \pm 5.42 \%)$ \\
\hline 97 & 133 & $1.05 \times 10^{+4}( \pm 4.66 \%)$ & $9.94 \times 10^{+3}( \pm 4.44 \%)$ & $8.87 \times 10^{+3}( \pm 5.48 \%)$ & $7.38 \times 10^{+3}( \pm 5.14 \%)$ & $5.79 \times 10^{+3}( \pm 5.85 \%)$ \\
\hline 133 & 174 & $2.42 \times 10^{+3}( \pm 5.02 \%)$ & $2.28 \times 10^{+3}( \pm 5.83 \%)$ & $2.00 \times 10^{+3}( \pm 5.45 \%)$ & $1.63 \times 10^{+3}( \pm 5.80 \%)$ & $1.22 \times 10^{+3}( \pm 6.05 \%)$ \\
\hline 174 & 220 & $6.59 \times 10^{+2}( \pm 5.65 \%)$ & $6.15 \times 10^{+2}( \pm 5.93 \%)$ & $5.32 \times 10^{+2}( \pm 4.82 \%)$ & $4.24 \times 10^{+2}( \pm 5.31 \%)$ & $3.05 \times 10^{+2}( \pm 7.15 \%)$ \\
\hline 220 & 272 & $2.02 \times 10^{+2}( \pm 5.26 \%)$ & $1.88 \times 10^{+2}( \pm 5.25 \%)$ & $1.60 \times 10^{+2}( \pm 4.75 \%)$ & $1.24 \times 10^{+2}( \pm 4.24 \%)$ & $8.37 \times 10^{+1}( \pm 4.44 \%)$ \\
\hline 272 & 330 & $6.76 \times 10^{+1}( \pm 4.80 \%)$ & $6.20 \times 10^{+1}( \pm 5.21 \%)$ & $5.21 \times 10^{+1}( \pm 5.07 \%)$ & $3.91 \times 10^{+1}( \pm 4.63 \%)$ & $2.43 \times 10^{+1}( \pm 5.70 \%)$ \\
\hline 330 & 395 & $2.40 \times 10^{+1}( \pm 4.98 \%)$ & $2.18 \times 10^{+1}( \pm 4.35 \%)$ & $1.80 \times 10^{+1}( \pm 4.90 \%)$ & $1.30 \times 10^{+1}( \pm 4.83 \%)$ & $7.23 \times 10^{+0}( \pm 5.90 \%)$ \\
\hline 395 & 468 & $8.92 \times 10^{+0}( \pm 4.87 \%)$ & $8.07 \times 10^{+0}( \pm 4.68 \%)$ & $6.50 \times 10^{+0}( \pm 3.62 \%)$ & $4.42 \times 10^{+0}( \pm 4.66 \%)$ & $2.12 \times 10^{+0}( \pm 6.01 \%)$ \\
\hline 468 & 548 & $3.43 \times 10^{+0}( \pm 4.11 \%)$ & $3.08 \times 10^{+0}( \pm 4.18 \%)$ & $2.42 \times 10^{+0}( \pm 4.23 \%)$ & $1.53 \times 10^{+0}( \pm 4.44 \%)$ & $6.05 \times 10^{-1}( \pm 6.08 \%)$ \\
\hline 548 & 638 & $1.37 \times 10^{+0}( \pm 4.23 \%)$ & $1.21 \times 10^{+0}( \pm 4.19 \%)$ & $9.19 \times 10^{-1}( \pm 4.46 \%)$ & $5.29 \times 10^{-1}( \pm 4.77 \%)$ & $1.63 \times 10^{-1}( \pm 6.91 \%)$ \\
\hline 638 & 737 & $5.53 \times 10^{-1}( \pm 4.43 \%)$ & $4.83 \times 10^{-1}( \pm 4.68 \%)$ & $3.50 \times 10^{-1}( \pm 4.83 \%)$ & $1.78 \times 10^{-1}( \pm 4.87 \%)$ & $4.04 \times 10^{-2}( \pm 8.56 \%)$ \\
\hline 737 & 846 & $2.28 \times 10^{-1}( \pm 5.00 \%)$ & $1.96 \times 10^{-1}( \pm 5.02 \%)$ & $1.34 \times 10^{-1}( \pm 5.30 \%)$ & $5.84 \times 10^{-2}( \pm 5.01 \%)$ & $8.86 \times 10^{-3}( \pm 9.05 \%)$ \\
\hline 846 & 967 & $9.48 \times 10^{-2}( \pm 5.16 \%)$ & $7.94 \times 10^{-2}( \pm 5.36 \%)$ & $5.06 \times 10^{-2}( \pm 5.38 \%)$ & $1.82 \times 10^{-2}( \pm 5.06 \%)$ & $1.65 \times 10^{-3}( \pm 11.51 \%)$ \\
\hline 967 & 1101 & $3.92 \times 10^{-2}( \pm 5.36 \%)$ & $3.20 \times 10^{-2}( \pm 5.65 \%)$ & $1.86 \times 10^{-2}( \pm 5.82 \%)$ & $5.24 \times 10^{-3}( \pm 5.36 \%)$ & $2.40 \times 10^{-4}( \pm 15.04 \%)$ \\
\hline 1101 & 1248 & $1.62 \times 10^{-2}( \pm 5.92 \%)$ & $1.27 \times 10^{-2}( \pm 5.94 \%)$ & $6.60 \times 10^{-3}( \pm 5.92 \%)$ & $1.37 \times 10^{-3}( \pm 5.87 \%)$ & $2.41 \times 10^{-5}( \pm 19.70 \%)$ \\
\hline 1248 & 1410 & $6.62 \times 10^{-3}( \pm 6.30 \%)$ & $4.97 \times 10^{-3}( \pm 6.35 \%)$ & $2.24 \times 10^{-3}( \pm 6.22 \%)$ & $3.14 \times 10^{-4}( \pm 6.73 \%)$ & $1.29 \times 10^{-6}( \pm 27.03 \%)$ \\
\hline 1410 & 1588 & $2.65 \times 10^{-3}( \pm 6.72 \%)$ & $1.88 \times 10^{-3}( \pm 6.81 \%)$ & $7.09 \times 10^{-4}( \pm 6.46 \%)$ & $6.05 \times 10^{-5}( \pm 8.09 \%)$ & $1.88 \times 10^{-8}( \pm 48.46 \%)$ \\
\hline 1588 & 1784 & $1.04 \times 10^{-3}( \pm 7.25 \%)$ & $6.83 \times 10^{-4}( \pm 7.25 \%)$ & $2.06 \times 10^{-4}( \pm 6.77 \%)$ & $9.17 \times 10^{-6}( \pm 10.21 \%)$ & $5.46 \times 10^{-12}( \pm 111.1 \%)$ \\
\hline 1784 & 2000 & $3.87 \times 10^{-4}( \pm 7.70 \%)$ & $2.34 \times 10^{-4}( \pm 7.62 \%)$ & $5.31 \times 10^{-5}( \pm 7.00 \%)$ & $9.53 \times 10^{-7}( \pm 12.01 \%)$ & - \\
\hline 2000 & 2238 & $1.37 \times 10^{-4}( \pm 8.16 \%)$ & $7.40 \times 10^{-5}( \pm 8.03 \%)$ & $1.17 \times 10^{-5}( \pm 7.22 \%)$ & $5.27 \times 10^{-8}( \pm 15.30 \%)$ & - \\
\hline 2238 & 2500 & $4.54 \times 10^{-5}( \pm 8.78 \%)$ & $2.12 \times 10^{-5}( \pm 8.55 \%)$ & $2.10 \times 10^{-6}( \pm 7.51 \%)$ & $8.65 \times 10^{-10}( \pm 23.54 \%)$ & - \\
\hline 2500 & 2787 & $1.37 \times 10^{-5}( \pm 9.36 \%)$ & $5.31 \times 10^{-6}( \pm 8.94 \%)$ & $2.85 \times 10^{-7}( \pm 8.16 \%)$ & $5.59 \times 10^{-13}( \pm 47.42 \%)$ & - \\
\hline 2787 & 3103 & $3.71 \times 10^{-6}( \pm 9.96 \%)$ & $1.13 \times 10^{-6}( \pm 9.57 \%)$ & $2.52 \times 10^{-8}( \pm 8.03 \%)$ & & - \\
\hline
\end{tabular}


and down by a factor of two, while the scale uncertainties at NLO + NLL have been computed as described in Sec. II. The values for the cross sections contain an additional error of $\mathcal{O}(1.5 \%)$ not shown explicitly from the numerical integration of the NLO corrections, which dominates both and is correlated between $\sigma_{\mathrm{NLO}}$ and $\sigma_{\mathrm{NLO}+\mathrm{NLL}}$. The PDF sets used and other parameters are given in the table captions. We also note that for the small $p_{T}$ bins $\left(p_{T} \lesssim 200 \mathrm{GeV}\right)$ at $\sqrt{S}=13 \mathrm{TeV}$, the threshold resummation may not be applicable anymore.
[1] R. Boughezal, C. Focke, X. Liu, and F. Petriello, Phys. Rev. Lett. 115, 062002 (2015).

[2] R. Boughezal, F. Caola, K. Melnikov, F. Petriello, and M. Schulze, Phys. Rev. Lett. 115, 082003 (2015).

[3] R. Boughezal, C. Focke, W. Giele, X. Liu, and F. Petriello, Phys. Lett. B 748, 5 (2015).

[4] X. Chen, T. Gehrmann, E. W. N. Glover, and M. Jaquier, Phys. Lett. B 740, 147 (2015).

[5] A. G.-D. Ridder, T. Gehrmann, E. W. N. Glover, A. Huss, and T. A. Morgan, Phys. Rev. Lett. 117, 022001 (2016).

[6] R. Boughezal, J. M. Campbell, R. K. Ellis, C. Focke, W. T. Giele, X. Liu, and F. Petriello, Phys. Rev. Lett. 116, 152001 (2016).

[7] J. Currie, E. W. N. Glover, and J. Pires, Phys. Rev. Lett. 118, 072002 (2017).

[8] J. Currie, A. G.-D. Ridder, T. Gehrmann, E. W. N. Glover, A. Huss, and J. Pires, Phys. Rev. Lett. 119, 152001 (2017).

[9] J. M. Campbell, R. K. Ellis, and C. Williams, Phys. Rev. Lett. 118, 222001 (2017).

[10] R. Boughezal, X. Liu, and F. Petriello, Phys. Lett. B 760, 6 (2016).

[11] J. M. Lindert et al., Eur. Phys. J. C 77, 829 (2017).

[12] R. Boughezal, A. Guffanti, F. Petriello, and M. Ubiali, J. High Energy Phys. 07 (2017) 130.

[13] B. Abelev et al. (ALICE Collaboration), Phys. Lett. B 722, 262 (2013).

[14] G. Aad et al. (ATLAS Collaboration), Eur. Phys. J. C 73, 2509 (2013).

[15] V. Khachatryan et al. (CMS Collaboration), Phys. Rev. C 96, 015202 (2017).

[16] G. Aad et al. (ATLAS Collaboration), J. High Energy Phys. 02 (2015) 154; 09 (2015) 141.

[17] S. Chatrchyan et al. (CMS Collaboration), Phys. Rev. D 90, 072006 (2014).

[18] M. Aaboud et al. (ATLAS Collaboration), J. High Energy Phys. 09 (2017) 020.

[19] V. Khachatryan et al. (CMS Collaboration), J. High Energy Phys. 03 (2017) 156.

[20] M. Aaboud et al. (ATLAS Collaboration), Phys. Rev. D 96, 072002 (2017).

[21] Tech. Rep. ATLAS-CONF-2017-048, CERN, Geneva, 2017, http://cds.cern.ch/record/2273864.

[22] V. Khachatryan et al. (CMS Collaboration), Eur. Phys. J. C 76, 451 (2016).

[23] D. Britzger, K. Rabbertz, D. Savoiu, G. Sieber, and M. Wobisch, arXiv:1712.00480.

[24] E. R. Nocera and M. Ubiali, arXiv:1709.09690.
[25] L. A. Harland-Lang, A. D. Martin, and R. S. Thorne, arXiv:1711.05757.

[26] J. Currie, E. W. N. Glover, T. Gehrmann, A. G.-D. Ridder, A. Huss, and J. Pires, Acta Phys. Pol. B 48, 955 (2017).

[27] X. Liu, S.-O. Moch, and F. Ringer, Phys. Rev. Lett. 119, 212001 (2017).

[28] N. Kidonakis, G. Oderda, and G. F. Sterman, Nucl. Phys. B525, 299 (1998).

[29] M. Dasgupta, F. Dreyer, G. P. Salam, and G. Soyez, J. High Energy Phys. 04 (2015) 039.

[30] Z.-B. Kang, F. Ringer, and I. Vitev, J. High Energy Phys. 10 (2016) 125.

[31] L. Dai, C. Kim, and A. K. Leibovich, Phys. Rev. D 94, 114023 (2016).

[32] N. Kidonakis and J. F. Owens, Phys. Rev. D 63, 054019 (2001).

[33] D. de Florian and W. Vogelsang, Phys. Rev. D 76, 074031 (2007).

[34] M. C. Kumar and S.-O. Moch, Phys. Lett. B 730, 122 (2014).

[35] D. de Florian, P. Hinderer, A. Mukherjee, F. Ringer, and W. Vogelsang, Phys. Rev. Lett. 112, 082001 (2014).

[36] M. Dasgupta, F. A. Dreyer, G. P. Salam, and G. Soyez, J. High Energy Phys. 06 (2016) 057.

[37] Z.-B. Kang, K. Lee, and F. Ringer, arXiv:1801.00790.

[38] A. Accardi, D. P. Anderle, and F. Ringer, Phys. Rev. D 91, 034008 (2015).

[39] M. Bonvini, S. Marzani, J. Rojo, L. Rottoli, M. Ubiali, R. D. Ball, V. Bertone, S. Carrazza, and N. P. Hartland, J. High Energy Phys. 09 (2015) 191.

[40] C. W. Bauer, S. Fleming, and M. E. Luke, Phys. Rev. D 63, 014006 (2000).

[41] C. W. Bauer, S. Fleming, D. Pirjol, and I. W. Stewart, Phys. Rev. D 63, 114020 (2001).

[42] C. W. Bauer and I. W. Stewart, Phys. Lett. B 516, 134 (2001).

[43] C. W. Bauer, D. Pirjol, and I. W. Stewart, Phys. Rev. D 65, 054022 (2002).

[44] M. Cacciari, G. P. Salam, and G. Soyez, J. High Energy Phys. 04 (2008) 063.

[45] T. Becher, M. Neubert, L. Rothen, and D. Y. Shao, Phys. Rev. Lett. 116, 192001 (2016).

[46] M. Dasgupta and G. P. Salam, Phys. Lett. B 512, 323 (2001).

[47] S. Caron-Huot, arXiv:1501.03754.

[48] A. J. Larkoski, I. Moult, and D. Neill, J. High Energy Phys. 09 (2015) 143. 
[49] A. J. Larkoski, I. Moult, and D. Neill, J. High Energy Phys. 11 (2016) 089.

[50] D. Neill, J. High Energy Phys. 01 (2017) 109.

[51] A. Broggio, A. Ferroglia, B. D. Pecjak, and Z. Zhang, J. High Energy Phys. 12 (2014) 005.

[52] T. Becher and M. Neubert, Phys. Lett. B 637, 251 (2006).

[53] T. Becher and G. Bell, Phys. Lett. B 695, 252 (2011).

[54] S. D. Ellis, C. K. Vermilion, J. R. Walsh, A. Hornig, and C. Lee, J. High Energy Phys. 11 (2010) 101.

[55] X. Liu and F. Petriello, Phys. Rev. D 87, 014018 (2013).

[56] Y.-T. Chien, A. Hornig, and C. Lee, Phys. Rev. D 93, 014033 (2016).

[57] L. Dai, C. Kim, and A. K. Leibovich, Phys. Rev. D 95, 074003 (2017).

[58] T. Becher, G. Bell, and S. Marti, J. High Energy Phys. 04 (2012) 034.

[59] R. Kelley, M. D. Schwartz, R. M. Schabinger, and H. X. Zhu, Phys. Rev. D 84, 045022 (2011).

[60] R. Boughezal, X. Liu, and F. Petriello, Phys. Rev. D 91, 094035 (2015).

[61] J. Gao, Z. Liang, D. E. Soper, H.-L. Lai, P. M. Nadolsky, and C.P. Yuan, Comput. Phys. Commun. 184, 1626 (2013).

[62] T. Becher and M. Neubert, Phys. Rev. Lett. 97, 082001 (2006).
[63] T. Becher and M. D. Schwartz, J. High Energy Phys. 02 (2010) 040.

[64] L. A. Harland-Lang, A. D. Martin, P. Motylinski, and R. S. Thorne, Eur. Phys. J. C 75, 204 (2015).

[65] Z. Nagy and D. E. Soper, arXiv:1711.02369.

[66] S. Catani, D. de Florian, M. Grazzini, and P. Nason, J. High Energy Phys. 07 (2003) 028.

[67] V. Ahrens, T. Becher, M. Neubert, and L. L. Yang, Eur. Phys. J. C 62, 333 (2009).

[68] S. Dulat, T. J. Hou, J. Gao, M. Guzzi, J. Huston, P. Nadolsky, J. Pumplin, C. Schmidt, D. Stump, and C. P. Yuan, Phys. Rev. D 93, 033006 (2016).

[69] B. Jäger, M. Stratmann, and W. Vogelsang, Phys. Rev. D 70, 034010 (2004).

[70] A. Mukherjee and W. Vogelsang, Phys. Rev. D 86, 094009 (2012).

[71] H.-L. Lai, M. Guzzi, J. Huston, Z. Li, P. M. Nadolsky, J. Pumplin, and C. P. Yuan, Phys. Rev. D 82, 074024 (2010).

[72] S. Alekhin, J. Blümlein, and S. Moch, arXiv:1803.07537.

[73] S. Alekhin, J. Blümlein, S. Moch, and R. Placakyte, Phys. Rev. D 96, 014011 (2017).

[74] H. Abramowicz et al. (ZEUS, H1 Collaborations), Eur. Phys. J. C 75, 580 (2015).

[75] R. D. Ball et al. (NNPDF Collaboration), Eur. Phys. J. C 77, 663 (2017). 